\title{
Dissecting cold gas in a high-redshift galaxy using a lensed background quasar ${ }^{\star}$
}

\author{
J.-K. Krogager ${ }^{1}$, P. Noterdaeme ${ }^{1}$, J. M. O’Meara ${ }^{2}$, M. Fumagalli ${ }^{3,4}$, J. P. U. Fynbo ${ }^{5}$, J. X. Prochaska ${ }^{6,7}$, \\ J. Hennawi ${ }^{8,9}$, S. Balashev ${ }^{10}$, F. Courbin ${ }^{11}$, M. Rafelski ${ }^{12,13}$, A. Smette ${ }^{14}$, and P. Boissé ${ }^{1}$ \\ ${ }^{1}$ Institut d'Astrophysique de Paris, CNRS-SU, UMR7095, 98bis bd Arago, 75014 Paris, France \\ e-mail: jens-kristian.krogager@iap.fr, krogager@iap.fr \\ 2 Department of Chemistry and Physics, Saint Michael's College, One Winooski Park, Colchester, VT 05439, USA \\ 3 Centre for Extragalactic Astronomy, Durham University, South Road, Durham, DH1 3LE, UK \\ ${ }^{4}$ Institute for Computational Cosmology, Durham University, South Road, Durham, DH1 3LE, UK \\ 5 The Cosmic Dawn Center, Niels Bohr Institute, University of Copenhagen, Juliane Maries Vej 30, 2100 Copenhagen Ø, Denmark \\ 6 Department of Astronomy and Astrophysics, University of California, 1156 High Street, Santa Cruz, CA 95064, USA \\ 7 University of California Observatories, Lick Observatory, 1156 High Street, Santa Cruz, CA 95064, USA \\ 8 Department of Physics, Broida Hall, University of California, Santa Barbara, CA 93106, USA \\ 9 Max-Planck-Institut für Astronomie, Königstuhl 17, 69117 Heidelberg, Germany \\ ${ }^{10}$ Ioffe Institute, Polytechnicheskaya ul. 26, Saint Petersburg 194021, Russia \\ 11 Institute of Physics, Laboratory of Astrophysics, École Polytechnique Fédérale de Lausanne (EPFL), Observatoire de Sauverny, \\ 1290 Versoix, Switzerland \\ 12 Space Telescope Science Institute, 3700 San Martin Drive, Baltimore, MD 21218, USA \\ 13 Department of Physics \& Astronomy, Johns Hopkins University, Baltimore, MD 21218, USA \\ 14 European Southern Observatory, Alonso de Córdova 3107, Vitacura, Santiago, Chile
}

Received 11 June 2018 / Accepted 31 August 2018

\begin{abstract}
We present a study of cold gas absorption from a damped Lyman- $\alpha$ absorber (DLA) at redshift $z_{\text {abs }}=1.946$ toward two lensed images of the quasar J144254.78+405535.5 at redshift $z_{\text {oso }}=2.590$. The physical separation of the two lines of sight at the absorber redshift is $d_{\mathrm{abs}}=0.7 \mathrm{kpc}$ according to our lens model. We observe absorption lines from neutral carbon and $\mathrm{H}_{2}$ along both lines of sight, indicating that cold gas is present on scales larger than $d_{\mathrm{abs}}$. We measure the column densities of $\mathrm{H}_{\mathrm{I}}$ to be $\log N\left(\mathrm{H}_{\mathrm{I}}\right)=20.27 \pm 0.02$ and $20.34 \pm 0.05$ and those of $\mathrm{H}_{2}$ to be $\log N\left(\mathrm{H}_{2}\right)=19.7 \pm 0.1$ and $19.9 \pm 0.2$. The metallicity inferred from sulphur is consistent with solar metallicity for both sightlines: $[\mathrm{S} / \mathrm{H}]_{\mathrm{A}}=0.0 \pm 0.1$ and $[\mathrm{S} / \mathrm{H}]_{\mathrm{B}}=-0.1 \pm 0.1$. Based on the excitation of low rotational levels of $\mathrm{H}_{2}$, we constrain the temperature of the cold gas phase to be $T=109 \pm 20$ and $T=89 \pm 25 \mathrm{~K}$ for the two lines of sight. From the relative excitation of fine-structure levels of $C_{\mathrm{I}}$, we constrain the hydrogen volumetric densities to lie in the range of $40-110 \mathrm{~cm}^{-3}$. Based on the ratio of observed column density and volumetric density, we infer the average individual "cloud" size along the line of sight to be $l \approx 0.1 \mathrm{pc}$. Using the transverse line-of-sight separation of $0.7 \mathrm{kpc}$ together with the individual cloud size, we are able to place an upper limit to the volume filling factor of cold gas of $f_{\mathrm{vol}}<0.1 \%$. Nonetheless, the projected covering fraction of cold gas must be large (close to unity) over scales of a few kpc in order to explain the presence of cold gas in both lines of sight. Compared to the typical extent of DLAs $(\sim 10-30 \mathrm{kpc})$, this is consistent with the relative incidence rate of $\mathrm{C}_{\mathrm{I}}$ absorbers and DLAs.
\end{abstract}

Key words. galaxies: high-redshift - cosmology: observations - quasars: absorption lines - gravitational lensing: strong galaxies: ISM

\section{Introduction}

The onset of star formation is intimately linked to the cooling and subsequent collapse of the neutral gas in galaxies. Under pressure equilibrium, the neutral gas naturally segregates into two distinct temperature phases: a cold $(T \sim 100 \mathrm{~K})$ neutral medium $(\mathrm{CNM})$, and a warm $\left(T \sim 10^{3}-10^{4} \mathrm{~K}\right)$ neutral medium (WNM), as characterized locally in the canonical twophase model (e.g., Field et al. 1969; Wolfire et al. 1995). In the distant Universe, the neutral gas phase is most readily accessible through observations of damped Ly $\alpha$ absorbers (DLAs;

\footnotetext{
* The reduced spectra (FITS files) are only available at the CDS via anonymous ftp to cdsarc.u-strasbg.fr (130.79.128.5) or via http://cdsarc.u-strasbg.fr/viz-bin/qcat?J/A+A/ $619 / \mathrm{A} 142$
}

Wolfe et al. 1986; Barnes et al. 2014), which make up the class of the $\operatorname{Ly} \alpha$ absorbers with the highest column density; they are defined as having $N_{\mathrm{HI}}>2 \times 10^{20} \mathrm{~cm}^{-2}$. However, as the presence and strength of Ly $\alpha$ absorption does not depend on temperature, the Ly $\alpha$ line alone does not constrain the relative contribution of CNM and WNM in DLAs. Since the interplay between the warm and cold neutral gas is crucial for the regulation of star formation, understanding how DLAs trace the CNM and WNM is therefore of great importance for galaxy evolution studies.

A direct way of probing the cold neutral gas at high redshift is through the use of H $21 \mathrm{~cm}$ absorption studies since its optical depth does depend on temperature (Gupta et al. 2009, 2012; Curran et al. 2010; Srianand et al. 2012; Kanekar et al. 2014; Dutta et al. 2017). Based on the spin-temperature measurements

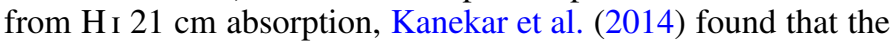


mass fraction of gas in DLAs with similar characteristics as the locally observed CNM must be $\lesssim 20 \%$, indicating that the CNM fraction is lower at high redshift than what is seen locally.

Another method for probing the physical conditions in DLAs is through the use of excited fine-structure levels of metal absorption lines, for instance, $\mathrm{C}_{\mathrm{I}}, \mathrm{C}_{\text {II }}$, and $\mathrm{Si}$ II (e.g., Wolfe et al. 2003, 2008; Howk et al. 2005; Srianand et al. 2005; Jorgenson et al. 2010; Neeleman et al. 2015). Since these finestructure transitions are mainly excited through collisions, the population ratio depends on density and temperature of the absorbing gas. For a large sample of DLAs, Neeleman et al. (2015) found that about 5\% show significant amounts of cold gas absorption. For the remaining absorbers, the physical properties are not well constrained because the modelling of finestructure levels is limited. The constraints are consistent with the gas being warm, however, similar to the metal-poor DLAs studied by Cooke et al. (2015), who inferred low densities ( $n \sim$ $\left.0.1 \mathrm{~cm}^{-3}\right)$ and high temperatures $(T \sim 5000 \mathrm{~K})$. Similar temperatures of $T \sim 10^{4} \mathrm{~K}$ for the warm gas phase are inferred directly from thermal Doppler broadening of ions with different masses (Carswell et al. 2012; Noterdaeme et al. 2012).

Molecular hydrogen provides a very useful and direct tracer of the CNM since its presence requires the gas to be cold and shielded from radiation (Krumholz et al. 2009; Krumholz 2012). Nevertheless, the Lyman and Werner absorption bands in the rest-frame ultraviolet fall in the Ly $\alpha$ forest, and it can be extremely challenging to detect these absorption lines, especially at low spectral resolution (Balashev et al. 2014). Targeted studies of $\mathrm{H}_{2}$ absorption for preselected DLAs showed that the fraction of DLAs with detectable amounts of $\mathrm{H}_{2}$ is very low, typically $\lesssim 10 \%$ (Petitjean et al. 2000; Ledoux et al. 2003; Noterdaeme et al. 2008; Jorgenson et al. 2014; Balashev \& Noterdaeme 2018).

Instead of relying on the $\mathrm{H}_{2}$ absorption lines as the main tracer of the CNM gas in DLAs, we can use absorption from $\mathrm{C}_{\mathrm{I}}$ as another proxy for the cold gas (Srianand et al. 2005; Jorgenson et al. 2010; Noterdaeme et al. 2018). Since the ionization potential of $\mathrm{C}_{\mathrm{I}}(11.3 \mathrm{eV})$ is lower than that of $\mathrm{H}_{\mathrm{I}}(13.6 \mathrm{eV})$, neutral carbon absorption arises only in highly shielded regions where the gas is able to cool efficiently. This makes $\mathrm{C}_{\mathrm{I}}$ an excellent tracer of the CNM (albeit limited to high-metallicity systems) and provides a direct probe of the cold gas phase. Moreover, the rest-frame transitions of the strongest absorption features from $\mathrm{C}_{\mathrm{I}}$ typically fall outside the Ly $\alpha$ forest, thereby providing the basis for an efficient selection of the CNM at high redshift (Ledoux et al. 2015).

While we can now directly trace the cold gas phase using proxies such as $\mathrm{C}_{\mathrm{I}}$ absorption, it is still not possible to obtain meaningful constraints on the filling factor of the cold gas since we only have one line of sight through the medium when using quasar absorbers. In a handful of cases, we are able to detect absorption systems in two lines of sight using closely projected quasar pairs (Hennawi et al. 2006, 2010; Prochaska et al. 2013; Rubin et al. 2015). However, such projected pairs typically probe large separations $d \gtrsim 10 \mathrm{kpc}$, and in no case has $\mathrm{C}_{\mathrm{I}}$ gas been observed in both sightlines.

If instead the background source is a strongly lensed quasar, the separation between the multiple lines of sight will change as a function of redshift, reaching a maximum separation at the lens redshift and converging at the source redshift. This provides a unique way of probing smaller physical separations $(\lesssim 5 \mathrm{kpc})$ if the absorption system is located between the source and the lens (e.g., Smette et al. 1995; Michalitsianos et al. 1997; Churchill et al. 2003; Ellison et al. 2007; Cooke et al. 2010).
While this has resulted in interesting constraints on the extent of the neutral gas phase (as probed by $\mathrm{HI}$ ), the cold gas properties on small scales remain unconstrained.

At lower redshifts $(z<1)$, three molecular, intervening absorption systems are known toward lensed radio quasars (Wiklind \& Combes 1995, 1996; Kanekar et al. 2005). In these cases, the absorption arises in the lens galaxies themselves and probes typical line-of-sight separations of $\sim 5 \mathrm{kpc}$. The absorption lines, detected at radio or submillimeter wavelengths, allow a detailed study of the kinematics and physical conditions in the lens galaxies. However, studying absorption in lensing galaxies at higher redshifts $(z \gtrsim 2)$ becomes increasingly difficult as high-redshift lens galaxies are extremely rare (Oguri \& Marshall 2010).

In this paper, we report the first detection of a high-redshift intervening absorber toward a lensed quasar at redshift $z_{\mathrm{em}}=$ 2.590 showing $\mathrm{C}_{\mathrm{I}}$ absorption in both lines of sight at redshift $z_{\mathrm{abs}}=1.946$. The absorber is not associated with the lensing galaxy as such a configuration would be unphysical given the quasar redshift and the observed image separation $(\theta=2.1 \operatorname{arcsec})$. The primary image (also the brightest, hereafter image A) of the quasar J144254.78+405535.5 (hereafter J1442+4055A) was selected through a direct search of $C_{\text {I }}$ absorbers in the Sloan Digital Sky Survey (as in the work by Ledoux et al. 2015). Through serendipitous inspection of the field, a nearby, unidentified point source with similar colors was targeted as a quasar pair candidate. Subsequent spectroscopic follow-up revealed the nearby point source to be a lensed image of the same quasar (hereafter image B). The quasar lens was simultaneously, but independently, discovered by a targeted search for quasar lens candidates (More et al. 2016; Sergeyev et al. 2016).

The position of the absorber in between the lens and the quasar means that the line-of-sight separation at $z=1.946$ is smaller than the maximum projected separation in the lens plane. We are therefore able to study the cold gas in this absorber over small transverse scales $(\approx 1 \mathrm{kpc})$. If the two sources had been a close pair instead of a lensed quasar, the physical separation at the absorber redshift would have been $\sim 17 \mathrm{kpc}$ instead. The small physical separation between the lines of sight enables us, for the first time, to place direct constraints on the volume filling factor of cold gas in a high-redshift, absorption-selected galaxy.

The paper is structured as follows: in Sect. 2 we describe the observations and data processing; in Sect. 3 we describe the lens model in order to obtain the line-of-sight separation at the absorber redshift; in Sect. 4 we describe the data analysis and measurements; and in Sect. 5 we present the interpretation of the derived observables and discuss our findings. Throughout this paper, we assume a flat $\Lambda \mathrm{CDM}$ cosmology with $\Omega_{\Lambda}=0.7$ and $H_{0}=68 \mathrm{~km} \mathrm{~s}^{-1} \mathrm{Mpc}^{-1}$ (Planck Collaboration XVI 2014).

\section{Observations and data processing}

The spectroscopic identification of the second quasar image J144254.60+405535.0 (hereafter J1442+4055B) was secured using the ALFOSC spectrograph mounted on the Nordic Optical Telescope (NOT) at the Observatorio Roque de los Muchachos, La Palma, Spain, through the fast-track program (observing ID: P52-415). The low-resolution spectroscopy using ALFOSC was obtained on April 28, 2016, using grism 4, which covers the whole optical wavelength range (3200-9600 $\AA$ ) with a spectral resolution of roughly $R=360$. The spectrum was taken using a slit-width of 1.3 arcsec during good conditions with a seeing of 1.0 arcsec. 
In order to search for emission from the lensing galaxy, we observed the field using the instrument ALFOSC in imaging mode to obtain a $r$-band image. The observations were carried out on August 3, 2016, using a nine-point dither pattern with individual integration times of $270 \mathrm{~s}$, resulting in a total integration time of $2430 \mathrm{~s}$. The images were bias subtracted using a median combined bias frame (based on 11 individual bias frames), and flat-field correction was performed using sky-flats obtained in twilight. Afterward, the images were background subtracted using the median sky value, and all images were subsequently shifted and combined into a single frame. The combined $r$-band image is shown in Fig. 1. We construct a model for the two quasar images using GALFIT (Peng et al. 2002) assuming that they are unresolved point sources using a combined point spread function from combined stars in the field. Moreover, we modeled the foreground galaxy located south of the lens images using a Sérsic profile (Sérsic 1963). We note that the lensing galaxy is included in the fit to avoid biasing the fit of the two quasar images. However, the model shown in Fig. 1 only includes the two quasar images and the foreground galaxy in order to highlight the lensing galaxy in the residual image (right-hand panel of Fig. 1). The lensing galaxy is clearly visible after subtracting the two point sources (see also Sergeyev et al. 2016).

The quasar lens was observed at low resolution using the LRIS spectrograph (Oke et al. 1995) at the Cassegrain focus of Keck-I on June 5, 2016. A slit-width of 1 arcsec was used, and the slit was oriented to cover both members of the pair simultaneously. The 560 dichroic, 600/7500 (0.8 $\mathrm{A} /$ pixel) grating, and $600 / 4000$ ( $0.6 \AA /$ pixel) grisms were used to provide continuous wavelength coverage from $3090-8651 \AA$. The data were flat fielded, optimally extracted, and flux calibrated (using the Feige 34 spectrophotometric standard star) with the LowRedux ${ }^{1}$ code.

The two quasar images were observed at high resolution using the HIRES spectrograph (Vogt et al. 1994) on May 20, 2017. Each observation was made using the $\mathrm{C} 1$ decker, which provides a spectral resolution of $\mathrm{R} \sim 48000$. The slit was aligned to have only one member of the quasar images in the slit at any one time. The choice of decker was made in part to further suppress contamination of one image by the other. The data span the wavelength range of $3029<\lambda<5880 \AA$. Quasar A was observed for at total of $5600 \mathrm{~s}$ over three integrations, and quasar B was observed for $7200 \mathrm{~s}$ over three integrations.

The data were reduced using the HIRedux ${ }^{2}$ code, which is a part of the $\mathrm{XIDL}^{3}$ package of astronomical routines. The data reduction and continuum fitting were performed in the same manner as presented in O'Meara et al. (2015).

In order to constrain the lensing galaxy in greater detail, we used the archival SDSS imaging data of the system in the five SDSS filters $(u, g, r, i$, and $z)$. We performed a similar modeling as described above for the NOT data using GALFIT to isolate the lensing galaxy. The lensing galaxy is undetected in the $u$ band, marginally detected in the $g$ band, but clearly detected in the $r$, $i$, and $z$ bands. However, since the image quality is worse than our deep NOT $r$ band, we used the structural parameters derived from the NOT data to constrain the fit to the SDSS data, that is, we kept the Sérsic index fixed to $n=4$, the axis ratio was fixed to 0.9 , and the half-light radius was fixed to $r_{\mathrm{e}}=3.2$ pixels. For the $g$ band, we used a slightly lower index of $n=3$ and

\footnotetext{
1 http://www. ucolick.org/ xavier/LowRedux/

2 http://www.ucolick.org/ xavier/HIRedux/

http://www . ucolick .org/ xavier/IDL/index .html
}

larger $r_{\mathrm{e}}=4$ pixels as galaxies tend to have lower indices and greater extent in the bluer rest-frame filters (Kelvin et al. 2012). The derived magnitudes in the detected filters are $g=21.0 \pm 0.6$, $r=19.3 \pm 0.1, i=18.7 \pm 0.1$, and $z=18.1 \pm 0.2$, all in the $\mathrm{AB}$ system. For comparison, the fainter quasar image $\mathrm{B}$ is similar in apparent magnitude, having an $r$-band magnitude of $r=19.0$, whereas image $\mathrm{A}$ is significantly brighter with $r=18.3$.

\section{Lens model}

\subsection{Lensing galaxy properties}

Based on the photometry from the SDSS images, we derive a photometric redshift estimate for the lensing galaxy using EAzy (Brammer et al. 2008). The derived photometric redshift is $z_{\text {phot }}=0.35 \pm 0.15$ based on the median of the posterior distribution. This estimate is highly uncertain because of the poor photometry and the few available filters, but it serves as a good initial guess for further analysis.

Figure 1 shows that the lensing galaxy is located closer to quasar image B than to image A. Based on the two LRIS spectra of images $\mathrm{A}$ and $\mathrm{B}$, where the slit was aligned to cover both images simultaneously in the slit, we therefore expect the lensing galaxy to contribute more to the spectrum of image B than to the spectrum of image A. We can quantify this expected contribution from the galaxy flux in the two spectra by using the NOT $r$-band image, since the NOT and LRIS observations were taken under similar seeing conditions. By imitating the slit orientation on top of the imaging data, we can calculate the amount of flux that would pass through a 1 arcsec slit. Applying the slit aperture to the $r$-band image results in a one-dimensional spatial profile that is consistent with what is observed in the LRIS data. Furthermore, since we have decomposed the $r$-band image into two quasar image contributions and lensing galaxy contribution, we can calculate the amount of galaxy flux expected in each spectrum. For spectrum A, the fraction of the total lensing galaxy flux is roughly $8 \%$, while for spectrum $\mathrm{B}$, the fraction of the total lensing galaxy flux is $32 \%$.

After constraining the relative amount of galaxy flux in the two spectra, we fit a combined quasar and lens galaxy model to the two spectra. For this purpose, we used the quasar template by Selsing et al. (2016) as a model for the intrinsic spectral shape of the quasar images. As a description of the lensing galaxy, we used the set of galaxy templates from Eazy generated with Pégase (Fioc \& Rocca-Volmerange 1997, see Brammer et al. (2008) for details describing the templates). Since EAZY had provided a best-fit template when the photometric redshift was estimated, we used this template as an initial guess. Since the cold-gas absorber at $z_{\mathrm{abs}}=1.946$ might harbor dust, which would redden the background quasars, we included a dust contribution at the absorber rest-frame to the model. We did not consider any contribution to the reddening of the quasar images from the lensing galaxy itself since we did not observe any significant absorption features from $\mathrm{Na}$ I and $\mathrm{Ca}$ II in the redshift range constrained from the posterior of $z_{\text {phot }}$. As the strength of $\mathrm{Na}$ I and $\mathrm{Ca}$ II is observed to correlate with dust reddening (Wild \& Hewett 2005; Murga et al. 2015), we rule out any significant reddening from the lensing galaxy at the projected position of the quasar images. Our assumption that the dust arises only in the high-redshift absorber is further bolstered by the fact that the best-fit values of $\mathrm{A}(\mathrm{V})$ are consistent with the inferred $\mathrm{A}(\mathrm{V})$ based on the depletion and metallicity (see Sect. 4.3). 

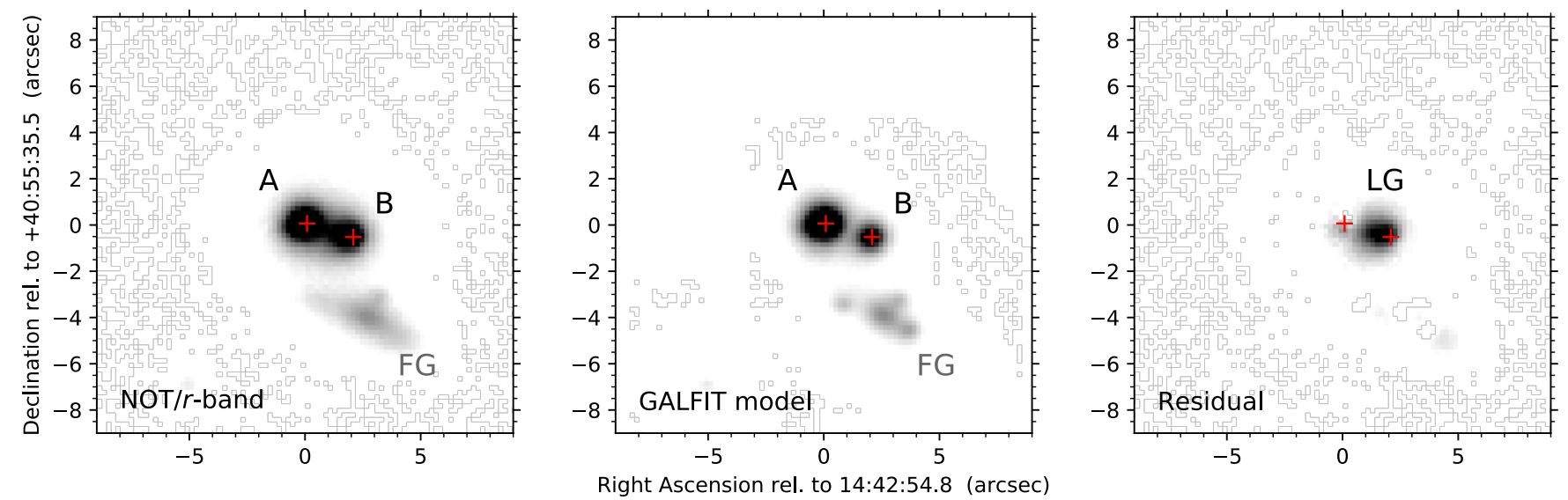

Fig. 1. Imaging of the field around the lensed quasar in $r$ band (left). Middle panel: model of the two quasar images (marked A and B) and an unrelated foreground galaxy (FG) using GALFIT (Peng et al. 2002). The centroids of the two quasar images are shown by red crosses in all frames. Right panel: residuals of the model-subtracted image, and the lensing galaxy (LG) is clearly visible. The same color scale is used for all frames.

The combined model for spectrum A (and similarly for B) is then given as

$f_{A}(\lambda)=r_{A} T_{\mathrm{QSO}}(\lambda) 10^{-0.4 \xi_{A}(\lambda) A(V)_{A}}+x_{A} r_{\mathrm{G}} T_{\text {gal }}\left(\lambda, z_{L}\right)$,

where $r_{A}$ denotes the absolute flux scaling of the quasar template, $T_{\mathrm{QSO}}, \xi_{A}$ denotes the reddening law in the rest-frame of the absorber toward quasar image $\mathrm{A}, \mathrm{A}(\mathrm{V})_{A}$ denotes the rest-frame $V$-band extinction, $x_{A}$ is the fraction of the total galaxy flux in spectrum $\mathrm{A}$, and $r_{\mathrm{G}}$ is the absolute flux scaling of the galaxy template, $T_{\mathrm{gal}}$, at lens redshift $z_{\mathrm{L}}$. Since the reddening law might be different along the two lines of sight, we included two separate terms $\xi_{A}$ and $\xi_{B}$ in the model. The two factors $x_{A}=0.08$ and $x_{B}=0.32$ were constrained from the imaging analysis. We fit both spectra and their ratio, $f_{A} / f_{B}$, simultaneously (as the ratio of the two adds additional constraints on the reddening and the galaxy spectrum).

We find that in both cases the spectra are well reproduced using the reddening law inferred for the Small Magellanic Cloud (SMC), but with a stronger $2175 \AA$ bump along sightline B. We parametrized the reddening law using the formalism of Fitzpatrick \& Massa (2007) and kept the parameters fixed to those of the average SMC reddening law except for parameter $c_{3}$ for sightline B, which controls the strength of the $2175 \AA$ bump.

Initially, we fixed the reddening law parameters and only varied $\mathrm{A}(\mathrm{V})_{A}, \mathrm{~A}(\mathrm{~V})_{B}, r_{A}, r_{B}, r_{\mathrm{G}}$, and $z_{\mathrm{L}}$. This provided a first estimate of the lens galaxy spectrum for the assumed galaxy template. We then fit the whole library of galaxy templates to the isolated lens galaxy spectrum in order to optimize the galaxy template. After converging on a galaxy template (of a single stellar population with ages of $\sim 11 \mathrm{Gyr}$ ), we fit the set of parameters again, this time also varying the bump strength along sightline B. Since the parameters are highly degenerate and the spectra might be affected by additional microlensing, we were unable to constrain the bump strength independently. Hence, we fixed the bump strength at $c_{3}=1.5$ for sightline $\mathrm{B}$, which corresponds to the bump strength observed for the LMC2 reddening law. This value of $c_{3}$ provides the best-fit lens galaxy spectrum.

The best-fit solution was obtained for the following parameters: $\mathrm{A}(\mathrm{V})_{A}=0.18 \pm 0.06 \mathrm{mag}, \mathrm{A}(\mathrm{V})_{B}=0.27 \pm 0.06 \mathrm{mag}$, and $z_{\mathrm{L}}=0.284 \pm 0.001$. The uncertainty on $\mathrm{A}(\mathrm{V})$ is here dominated by the systematic uncertainty related to the unknown intrinsic shape of the quasar spectrum. From the observed dispersion of quasar power-law shapes (Krawczyk et al. 2015), we obtain the systematic $1 \sigma$ uncertainty of $0.06 \mathrm{mag}$. The LRIS data together with the best-fit model are shown in Fig. 2, and the resulting isolated lens galaxy spectrum together with the best-fit template is shown in Fig. 3. The lensing galaxy spectrum and the best-fit template were corrected for slit loss ${ }^{4}$. In Fig. 3 we also show the SDSS photometry in $g, r$, and $i$. Because the galaxy is an extended source, only $40 \%$ of the total flux makes it into the 1 arcsec slit. Overall, the rescaled photometry is fully consistent with the inferred lens galaxy spectrum.

The spectral region from $\sim 5000-7000 \AA$ might be affected by variations in the broad emission lines together with the iron pseudo-continuum from a blend of many Fe II and Fe III emission lines. Nonetheless, the absorption features from the Ca II, Mg I, and $\mathrm{Na}$ I lines as well as the $4000 \AA$ break in the galaxy spectrum strongly constrain the lens redshift.

The $A(V)$ measurements are not significantly affected by the bump strength (the variations are about $0.01 \mathrm{mag}$, less than the systematic uncertainty due to quasar continuum shape), but we note that the reddening law cannot be constrained independently since the shape of the reddening law is degenerate with the galaxy and quasar template shapes. This is further complicated by a possible microlensing effect of the quasar continuum. Although microlensing in general is achromatic, it can lead to chromatic effects if the continuum-emitting region has a radial color gradient, as is expected for most accretion disk models (e.g., Wambsganss \& Paczynski 1991). However, with only one epoch of observations, these effects are impossible to disentangle.

\subsection{Line-of-sight separation}

We calculated the distance between the two quasar sightlines as a function of redshift from $z=0$ to the redshift of the quasar following Smette et al. (1992). A simplified geometry is illustrated in Fig. 4.

The angle on the sky between the two images is denoted $\theta$, the distance between the two sightlines is denoted $\mathrm{d}(z)$, and the angular diameter distances between source and lens, source and

\footnotetext{
4 Based on the simulated slit aperture of the imaging data, we calculate that only $40 \%$ of the galaxy flux falls within the 1 arcsec slit, of which $80 \%$ is included in the extraction aperture of spectrum B.
} 

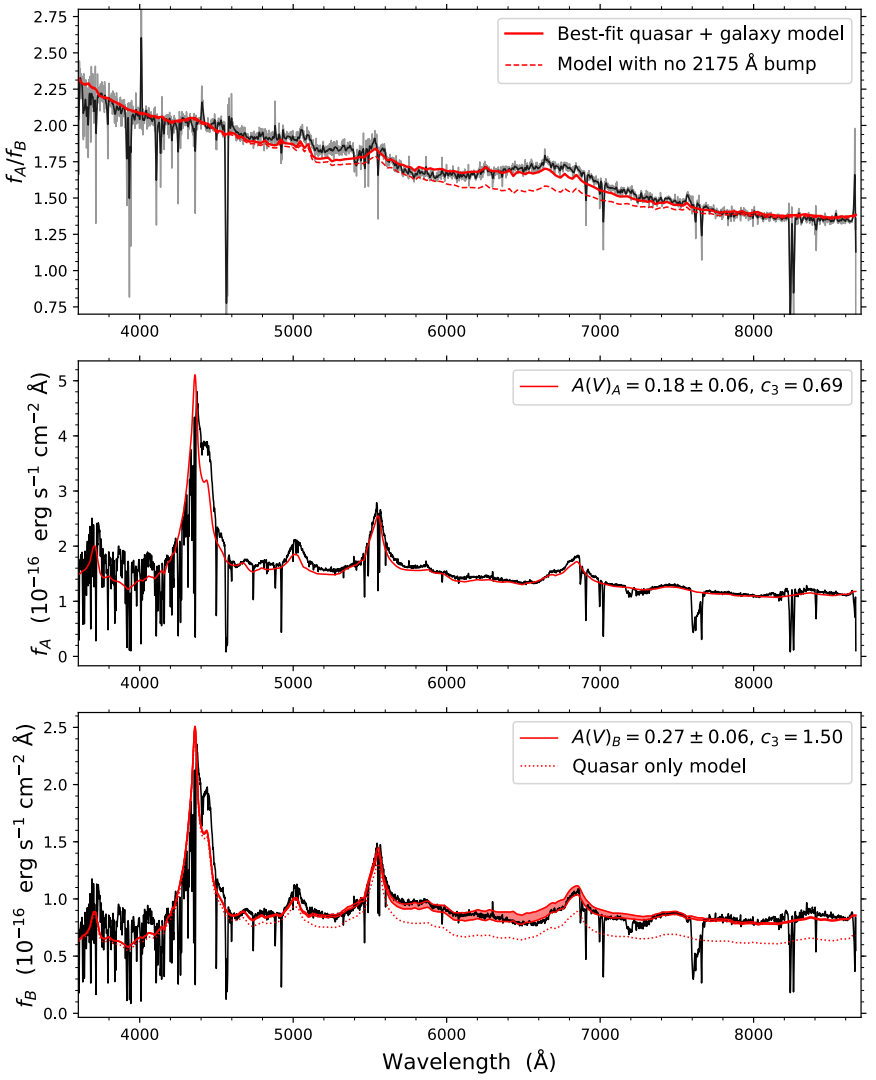

Fig. 2. Spectra for the two lines of sight using the Keck/LRIS data. In the top panel, we show the ratio of the two spectra together with the best-fit quasar and galaxy model in red (see text). For comparison, we show the same model without the $2175 \AA$ bump. In the middle and bottom panels, we show the individual spectra together with their bestfit dust-reddened quasar plus galaxy model in red. The shaded region in the bottom panel indicates the location and strength of the $2175 \AA$ dust bump. The dotted line in the bottom panel shows the quasar-only contribution to the model.

absorber, and observer and lens are denoted $D_{\mathrm{SL}}, D_{\mathrm{SA}}$, and $D_{\mathrm{OL}}$, respectively. From Smette et al. (1992), we calculate d(z) as

$\mathrm{d}(z)=\theta \frac{D_{\mathrm{SA}} D_{\mathrm{OL}}}{D_{\mathrm{SL}}}$

We then calculated the line-of-sight separation as a function of redshift for the case of J1442+4055 assuming a lens redshift of $z_{\mathrm{L}}=0.284$ and a source redshift of $z_{\mathrm{S}}=2.590$. From imaging of the field obtained at the NOT (see Fig. 1), we find $\theta=2.13 \pm$ $0.01 \mathrm{arcsec}$. The resulting line-of-sight separation as a function of redshift is shown in Fig. 5, and at the redshift of the absorber, $z_{\mathrm{abs}}=1.946$, we find $d_{\mathrm{abs}}=0.71 \pm 0.01 \mathrm{kpc}$ for the best-fit lens redshift of $z_{\mathrm{L}}=0.284 \pm 0.001$.

\section{Results}

\subsection{Metal absorption}

We detect a large number of absorption lines from metal species in the high-resolution spectra at a redshift of $z_{\mathrm{abs}}=1.946938$ from various ionization stages: $\mathrm{C}_{\text {I }}, \mathrm{O}_{\text {I, }} \mathrm{Si}$ II, Fe II, S II, Ni II, Al II, $\mathrm{Al}$ III, C IV, and Si IV.

In order to obtain column densities of the singly ionized species (which is the dominant ionization state for metals in

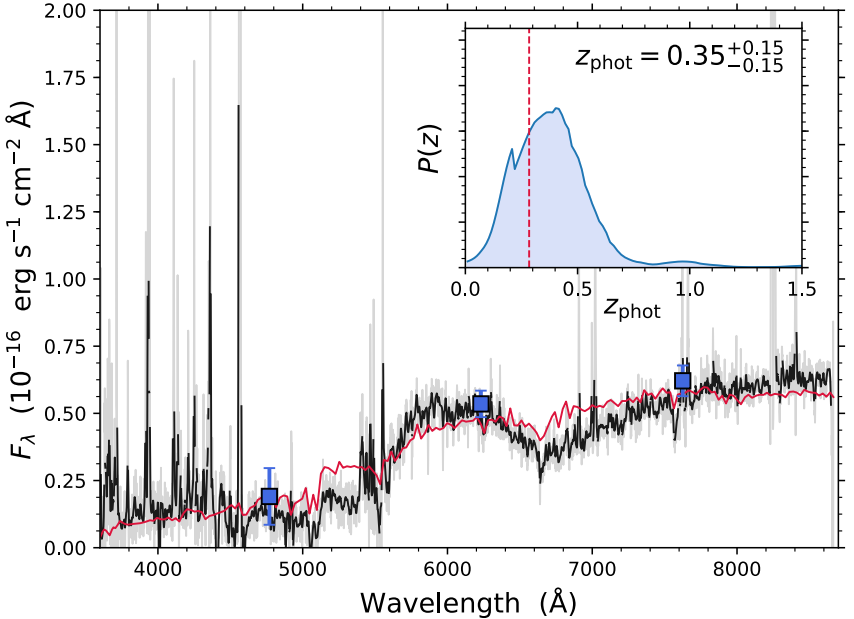

Fig. 3. Isolated lens galaxy spectrum recovered from spectral fitting. The light gray line shows the raw spectrum, and in black we show the Gaussian-filtered spectrum with a median clipping to remove outlying pixels caused by variations in absorption lines from the $z_{\text {abs }}=1.946$ system. The red line indicates the best-fit galaxy template of a single stellar population $(t=11 \mathrm{Gyr})$, and the blue squares show the SDSS photometry of the lensing galaxy. The inset in the upper right corner shows the posterior probability of the photometric redshift estimation from EAZY, where the dashed red line marks the best-fit lens redshift.

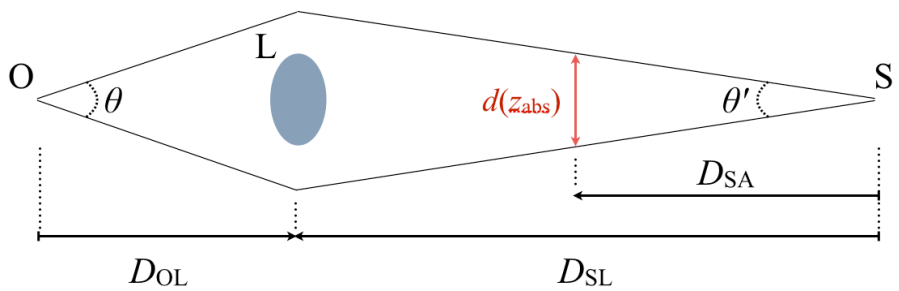

Fig. 4. Lensing geometry. The distances between observer (O), lens (L), source (S), and absorber (A) at redshift $z_{\text {abs }}$ are illustrated.

the neutral gas), we fit the transitions of Si II $\lambda \lambda 1304,1808$, Fe II $\lambda \lambda 1608,1611, S_{\text {II }} \lambda \lambda 1250$, and 1253 using Voigt profiles implemented in the Python module VoigtFit (Krogager 2018) with a total of eight and six components for lines of sight $\mathrm{A}$ and B, respectively. We assumed that the redshifts and Dopplerbroadening parameters of each components of the three heavy ions are identical. Thus the different $z$ and $b$ parameters for the components of S, Si, and Fe are tied during the fit. In Table 1, we give the total column densities derived for the two lines of sight. The best-fit parameters for the individual components are summarized in Appendix A together with figures showing the best-fit line profiles.

We furthermore fit the following absorption lines from $\mathrm{C}_{\mathrm{I}}$ fine-structure transitions: $C_{\text {I }} \lambda 1280, \lambda 1560$, and $\lambda 1656$. Since the individual velocity components are barely resolved, we assumed that the excited fine-structure levels, $J=1\left(\mathrm{CI}^{*}\right)$ and $J=2$ $\left(\mathrm{C}^{* *}\right)$, follow the same kinematic structure as the ground-level $(J=0)$, that is, redshifts and broadening parameters for individual components are tied during the fit. Moreover, because of the low signal-to-noise ratio, we assumed a constant column density ratio of $\mathrm{C}_{\mathrm{I}^{*}}$ to $\mathrm{C}_{\mathrm{I}}\left(r^{*}\right)$ and $\mathrm{C}_{\mathrm{I}}{ }^{* *}$ to $\mathrm{C}_{\mathrm{I}}\left(r^{* *}\right)$ for all components. The total column densities derived for $\mathrm{C}_{\mathrm{I}}$ and the column density ratios of the excited levels with respect to the ground level are summarized in Table 1. We identify two weak, intervening 


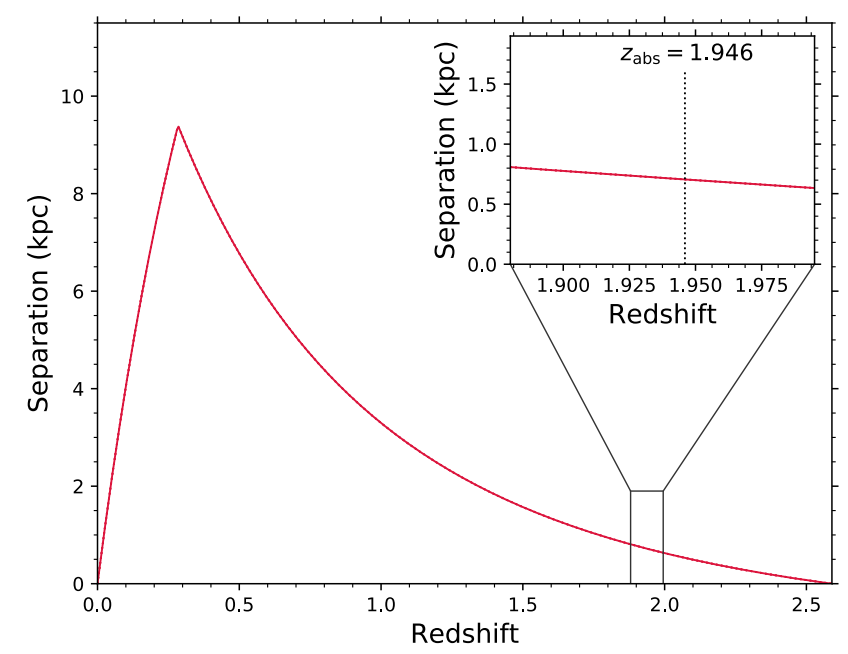

Fig. 5. Distance between the two sightlines as a function of redshift assuming a lens redshift of $z_{\mathrm{L}}=0.284$.

Table 1. Overview of measurements.

\begin{tabular}{lcc}
\hline \hline Observable & LOS-A & LOS-B \\
\hline $\log \mathrm{N}\left(\mathrm{HI}_{\mathrm{I}}\right)$ & $20.27 \pm 0.02$ & $20.34 \pm 0.05$ \\
$\log \mathrm{N}\left(\mathrm{H}_{2}\right)$ & $19.7 \pm 0.1$ & $19.9 \pm 0.2$ \\
$\log \mathrm{N}(\mathrm{H})$ & $20.4 \pm 0.1$ & $20.6 \pm 0.1$ \\
$f_{\mathrm{H}_{2}}$ & $0.3 \pm 0.1$ & $0.4 \pm 0.1$ \\
$\log \mathrm{N}(\mathrm{Fe})$ & $14.93 \pm 0.03$ & $14.80 \pm 0.03$ \\
$\log \mathrm{N}(\mathrm{Si})$ & $15.55 \pm 0.03$ & $15.37 \pm 0.06$ \\
$\log \mathrm{N}(\mathrm{S})$ & $15.52 \pm 0.03$ & $15.63 \pm 0.07$ \\
{$[\mathrm{Fe} / \mathrm{S}]$} & $-1.0 \pm 0.1$ & $-1.2 \pm 0.1$ \\
{$[\mathrm{Si} / \mathrm{S}]$} & $-0.4 \pm 0.1$ & $-0.7 \pm 0.1$ \\
$A_{\mathrm{V}}$ & $0.19 \pm 0.02$ & $0.36 \pm 0.02$ \\
$\log \mathrm{N}(\mathrm{C} \mathrm{I})$ & $14.25 \pm 0.02$ & $14.79 \pm 0.05$ \\
$\log r^{*}$ & $-0.40 \pm 0.04$ & $-1.18 \pm 0.13$ \\
$\log r^{* *}$ & $-0.72 \pm 0.07$ & $-1.65 \pm 0.14$ \\
\hline
\end{tabular}

Notes. All column densities are given in units of $\mathrm{cm}^{-2}$.

$\mathrm{C}$ IV absorbers toward sightline $\mathrm{A}$ and one weak, intervening $\mathrm{C}_{\text {IV }}$ absorber toward sightline $\mathrm{B}$. They overlap with the $\mathrm{C}_{\mathrm{I}}$ line complexes and were included in the fit in order to obtain a good fit. The best-fit parameters for the $\mathrm{C}$ I and $\mathrm{C}$ IV components included in the models are summarized in Appendix A together with a figure showing the individual lines we used to constrain the fits. Moreover, we observe two $\mathrm{C}$ IV absorption systems along the two lines of sight at redshifts $z=2.5861$ (intrinsic to the quasar) and $z=2.1179$.

\subsection{Atomic and molecular hydrogen}

We observe an asymmetry in the red wing of the Ly $\alpha$ line profiles for both sightlines, but it is more pronounced for sightline B, see Fig. 6. In order to properly model the absorption lines, we therefore fit a multi-component model to the Ly $\alpha$ line. For both lines of sight, the first component was fixed to the average redshift of the low-ionization metals weighted by column density. This "bulk" component properly reproduces the blue wing of the Ly $\alpha$ profile in both sightlines.

The red wing was in turn fit by adding a component to match the asymmetry. Similarly to the analysis by Noterdaeme et al. (2008), the redder components required quite high Doppler

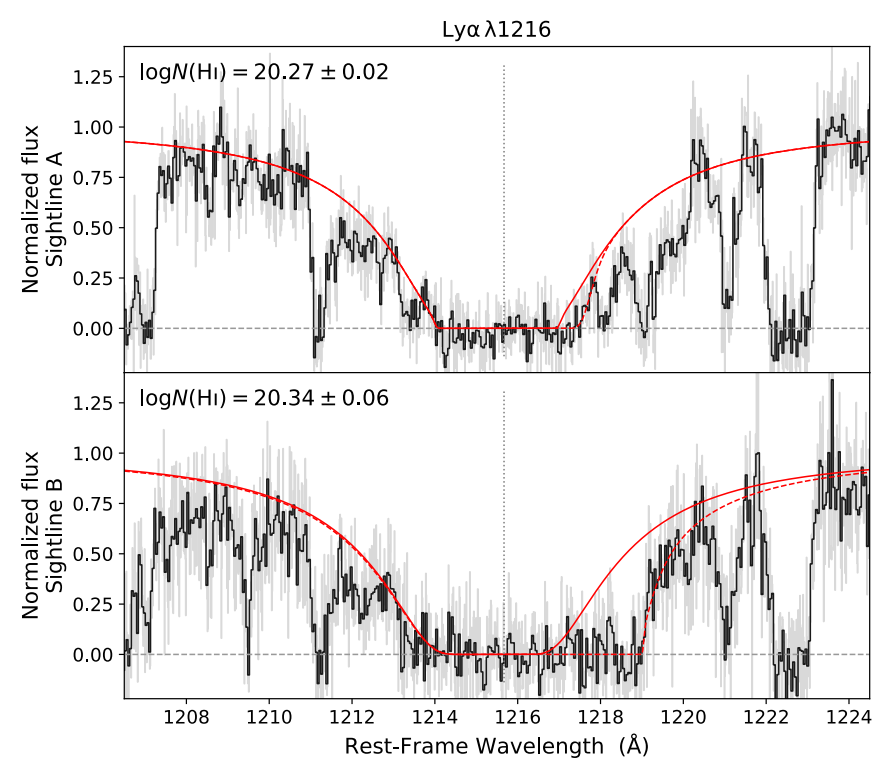

Fig. 6. Comparison of the Ly $\alpha$ absorption profiles for sightlines A (top) and B (bottom) in the rest-frame of the absorber $\left(z_{\text {sys }}=1.946938\right)$ from the Keck/HIRES spectra. The data have been rebinned for visual clarity (black line), and we show the unbinned data as the thin gray line for comparison. The total best-fit profile is shown as the dashed red line. The solid red line shows the H I profile for the bulk of the metal absorption. The dotted vertical line marks the resonant wavelength in the systemic rest-frame.

broadening in order to fit the sharp edge of the red wing. For sightline B, where the asymmetry is more pronounced, we needed to include two red components in order to match the profile well. The best-fit column densities for the H I component associated with the low-ionization metals are summarized in Table 1. We did not observe any significant absorption from singly ionized species at the relative velocity of the redder components. However, the higher ionization lines, for example, $\mathrm{C}$ IV and $\mathrm{Al}$ III, do show absorption at similar relative velocities. We therefore argue that this gas phase has a higher ionization fraction and is not directly related to the gas carrying the bulk of the metals.

Moreover, we detect absorption lines from the Lyman-bands of $\mathrm{H}_{2}, \mathrm{~B}^{1} \Sigma_{\mathrm{u}}^{+}(v) \leftarrow \mathrm{X}^{1} \Sigma_{\mathrm{g}}^{+}(v=0)$ for $v$ up to $v=4$ for sightline A. The data for sightline $\mathrm{B}$ are noisier, therefore we only recovered the first four bands up to $v=3$. We fit rotational levels up to $J=3$ for both sightlines, using one component for each $J$ level. Since the lines are damped, we were unable to constrain the $b$ parameter. We therefore performed a first fit with an arbitrary, fixed $b$ parameter of $b=5 \mathrm{~km} \mathrm{~s}^{-1}$ in order to obtain the redshift of the best-fit components. In both cases, the best-fit redshift corresponds to a component of $\mathrm{C}_{\mathrm{I}}$, and we therefore tied the redshift and $b$ parameter to those of the matching $\mathrm{C}_{\mathrm{I}}$ component for each sightline. This agreement between $\mathrm{C}_{\mathrm{I}}$ and $\mathrm{H}_{2}$ components is consistent with the tight observed relationship between $\mathrm{C}_{\mathrm{I}}$ and $\mathrm{H}_{2}$ (Noterdaeme et al. 2018). We assumed the same $b$ parameter for all $J$ levels and neglected any additional thermal contribution to the broadening due to the low temperature observed $(T \sim 100 \mathrm{~K})$, see Sect. 5.1. The observed spectra together with the best-fit models are shown in Fig. 7, and the best-fit parameters are summarized in Appendix A. The molecular bands are highly blended with the Ly $\alpha$ forest and the low signal-to-noise ratio makes it very difficult to constrain all the molecular transitions, especially for sightline B. The tightest constraints to the fit come from the $v=0$ and $v=1$ bands. 

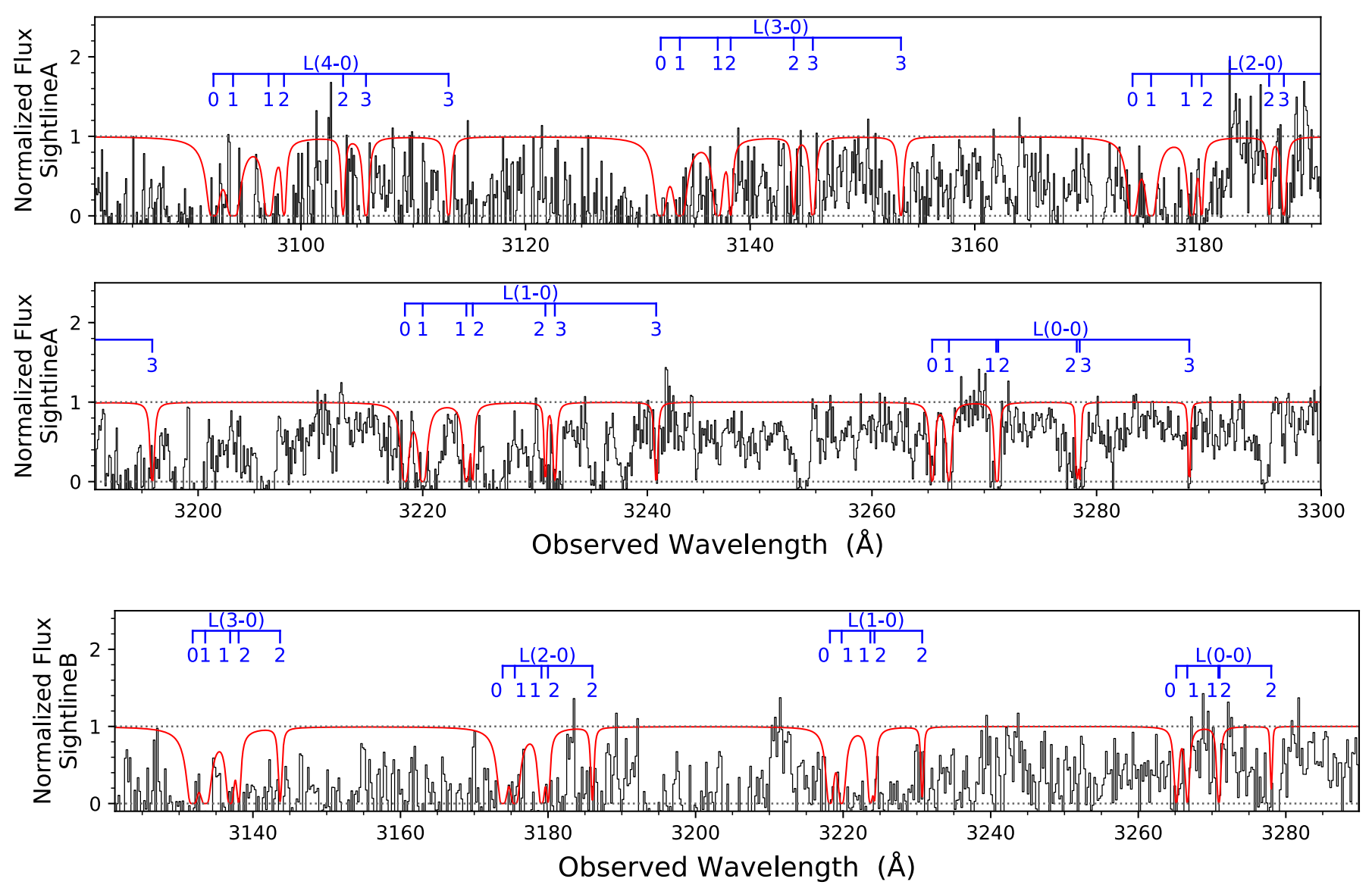

Fig. 7. Molecular absorption bands of $\mathrm{H}_{2}$ for sightlines A (top two panels) and B (bottom panel) from the Keck/HIRES spectra. The locations of the rotational $J$ level for each of the vibrational bands are shown in blue above the spectra. The best-fit absorption profiles are shown in red. The data have been rebinned and smoothed (using Gaussian smoothing with an FWHM of 1.5 pixels) for visual clarity.

Last, since the data are relatively noisy, the fit was performed independently by two individuals of our team using two different fitting software packages, and the two results are consistent within the rather large uncertainty $(\sim 0.1-0.2 \mathrm{dex})$.

\subsection{Metallicity}

Using the total hydrogen column density $N(\mathrm{H})=N\left(\mathrm{H}_{\mathrm{I}}\right)+$ $2 N\left(\mathrm{H}_{2}\right), \log N(\mathrm{H})=20.4 \pm 0.1$ and $20.6 \pm 0.1$ for sightlines $\mathrm{A}$ and $\mathrm{B}$, respectively, we derived an average observed metallicity for the two lines of sight using sulphur since this element does not significantly deplete into dust grains (Savage \& Sembach 1996). For sightlines $\mathrm{A}$ and $\mathrm{B}$, we find $[\mathrm{S} / \mathrm{H}]_{A}=0.0 \pm 0.1$ and $[\mathrm{S} / \mathrm{H}]_{B}=-0.1 \pm 0.1$.

Since the observed column density ratio of $\mathrm{S}$ to $\mathrm{Fe}$ indicates significant amounts of dust in the gas, we considered dust corrections to the metallicities ${ }^{5}$. We used the depletion sequences as determined by De Cia et al. (2016) in order to calculate the fraction of metals locked up in dust grains. The formalism by De Cia et al. (2016) uses $[\mathrm{Zn} / \mathrm{Fe}]$ as the overall tracer of the depletion sequences, but as our high-resolution spectra do not cover the zinc lines at $\lambda 2026$ and $\lambda 2062$, we used $[\mathrm{S} / \mathrm{Fe}]$ as a tracer of $[\mathrm{Zn} / \mathrm{Fe}]$ since both elements deplete very little into dust. Moreover, Berg et al. (2015) reported $[\mathrm{S} / \mathrm{Zn}] \approx 0$ for high-

\footnotetext{
5 We note that nucleosynthetic effects may play a role in the $[\mathrm{S} / \mathrm{Fe}]$ ratio, but at solar metallicity, the enhancement of $\alpha$-elements is likely negligible (e.g., Caffau et al. 2005; Berg et al. 2015).
}

metallicity DLAs (Rafelski et al. 2012; De Cia et al. 2016, see also). Using Eq. (5) from the work by De Cia et al. (2016), we obtained consistent dust-corrected metallicities for both sightlines, using both iron and sulphur, of $[\mathrm{Fe} / \mathrm{H}]_{0}=0.2 \pm 0.1$ and $[\mathrm{S} / \mathrm{H}]_{0}=0.3 \pm 0.1$, respectively.

Using the depletion sequences of De Cia et al. (2016), we furthermore calculated the expected optical extinction $\mathrm{A}(\mathrm{V})$ based on the observed metallicity and hydrogen column density. For the two sightlines $A$ and $B$, we then infer a rest-frame $A(V)$ of $0.2 \pm 0.1$ and $0.4 \pm 0.1$, respectively. We note that to calculate $\mathrm{A}(\mathrm{V})$, we used the total hydrogen column density, $\log N(\mathrm{H})$, instead of just the atomic hydrogen column density $\log N(\mathrm{H} \mathrm{I})$, which is used in Eq. (8) by De Cia et al. (2016).

\section{Discussion}

\subsection{Physical properties}

Using the derived column densities of $\mathrm{H}_{2}$ for the two lowest rotational levels $(J=0$ and $J=1)$, we can infer the excitation temperature, $T_{01}$, which is a good proxy for the overall kinetic temperature of the molecular medium (Roy et al. 2006). For both sightlines, we infer consistent temperatures of $T_{01}^{A}=109 \pm 20 \mathrm{~K}$ and $T_{01}^{B}=89 \pm 25 \mathrm{~K}$.

The $\mathrm{C}_{\text {I }}$ fine-structure levels can be excited either by collisions (in high-density or shielded gas) or by UV pumping from the incident UV flux (in low-density gas). However, the incident radiation field required to excite $\mathrm{C}_{\mathrm{I}}$ to the observed levels 
for $\mathrm{J} 1442+4055$ is roughly 30 times higher than the Galactic UV field (Habing 1968). We therefore neglect any contribution from the incident UV field in the following calculations, since the high amount of dust extinction indicates that the gas is efficiently shielded from UV photons ${ }^{6}$. We can then constrain the density of the cold gas phase assuming the temperature measurements derived from $\mathrm{H}_{2}$ above. We used the code Popratio (Silva \& Viegas 2001) to calculate the expected population of the excited fine-structure levels compared to the ground-state population in a grid of temperature and hydrogen density, $n_{\mathrm{H}}$. For this calculation, we included a contribution from the cosmic microwave background at the absorber redshift and from the extragalactic UV background field (Khaire \& Srianand 2018) ${ }^{7}$. Since most of the neutral hydrogen is likely not directly associated with the cold gas from which the bulk of the $\mathrm{C}_{\mathrm{I}}$ absorption arises, we argue that the molecular fraction in the cold gas phase is higher than the average $f_{\mathrm{H}_{2}}$ and probably closer to unity, that is, the $\mathrm{C}_{\mathrm{I}}$ absorption arises predominantly from fully molecular gas (Bialy \& Sternberg 2016, e.g.,). Hence, we ran the PopRatio models assuming $f_{\mathrm{H}_{2}}=1$. The constraints from the observed ratios of $N\left(\mathrm{CI}^{*}\right) / \mathrm{N}\left(\mathrm{C}_{\mathrm{I}}\right)$ and $N\left(\mathrm{C}_{\mathrm{I}^{* *}}\right) / \mathrm{N}\left(\mathrm{C}_{\mathrm{I}}\right)$ are shown in Fig. 8. We subsequently took into account the temperature constraints from $\mathrm{H}_{2}$ excitation (see the shaded regions in Fig. 8) and marginalized the two-dimensional probability distribution over $T$ in order to obtain the most probable density (right-hand panels of Fig. 8). The estimated hydrogen densities for sightlines $\mathrm{A}$ and $\mathrm{B}$ are $n_{\mathrm{H}}^{\mathrm{A}} \approx 110 \mathrm{~cm}^{-3}$ and $n_{\mathrm{H}}^{\mathrm{B}} \approx 40 \mathrm{~cm}^{-3}$.

For comparison, we ran a set of models using the average observed $f_{\mathrm{H}_{2}}$ integrated over the full line of sight (see Table 1). For the lower molecular fraction, we obtain lower densities by a factor of 2.2 (see the dotted lines in the right-hand panels of Fig. 8). However, we caution that these estimates are strict lower limits, as the molecular fraction inside the cold medium is certainly larger than the average integrated molecular fraction.

\subsection{Kinematics}

The low-ionization species (e.g., Si II and Fe II) for the two different lines of sight show absorption over very similar velocity spreads $\Delta v \sim 200 \mathrm{~km} \mathrm{~s}^{-1}$ with several subcomponents. The individual components show different strengths, but the absorption is dominated by a strong red component for sightline A, defined as the systemic redshift. In contrast, sightline B exhibits stronger absorption in the blueshifted component at $v_{\text {rel }}=-100 \mathrm{~km} \mathrm{~s}^{-1}$. A similar pattern is observed in the higher ionization lines of Al III. While the neutral carbon absorption is concentrated over a smaller range in velocity space than the low-ionization species, the main component of $\mathrm{C}_{\mathrm{I}}$ absorption corresponds to the strongest Fe II component for both lines of sight (see Fig. 9). There is a significant velocity shift for $\mathrm{C}_{\mathrm{I}}$ between the two sightlines of $\Delta v_{\mathrm{C}_{\mathrm{I}}} \approx 100 \mathrm{~km} \mathrm{~s}^{-1}$. This fits a scenario well in which the low-ionization species predominantly arise in a warmer diffuse phase (similar to the warm neutral medium of the Milky Way) spread over a larger velocity width. The cold gas clumps are more confined in velocity space, likely tracing a more spatially confined structure as well. If we interpret this velocity offset as a consequence of ordered rotation, we can use the observed velocity shift as an indicator of the dynamical mass of the system.

\footnotetext{
6 We caution that the dust reddening might not be fully associated with the cold gas phase.

7 We have implemented the updated calculation of the extragalactic UV background from Khaire \& Srianand (2018) in PopraTIo instead of the default background field by Madau et al. (1999).
}

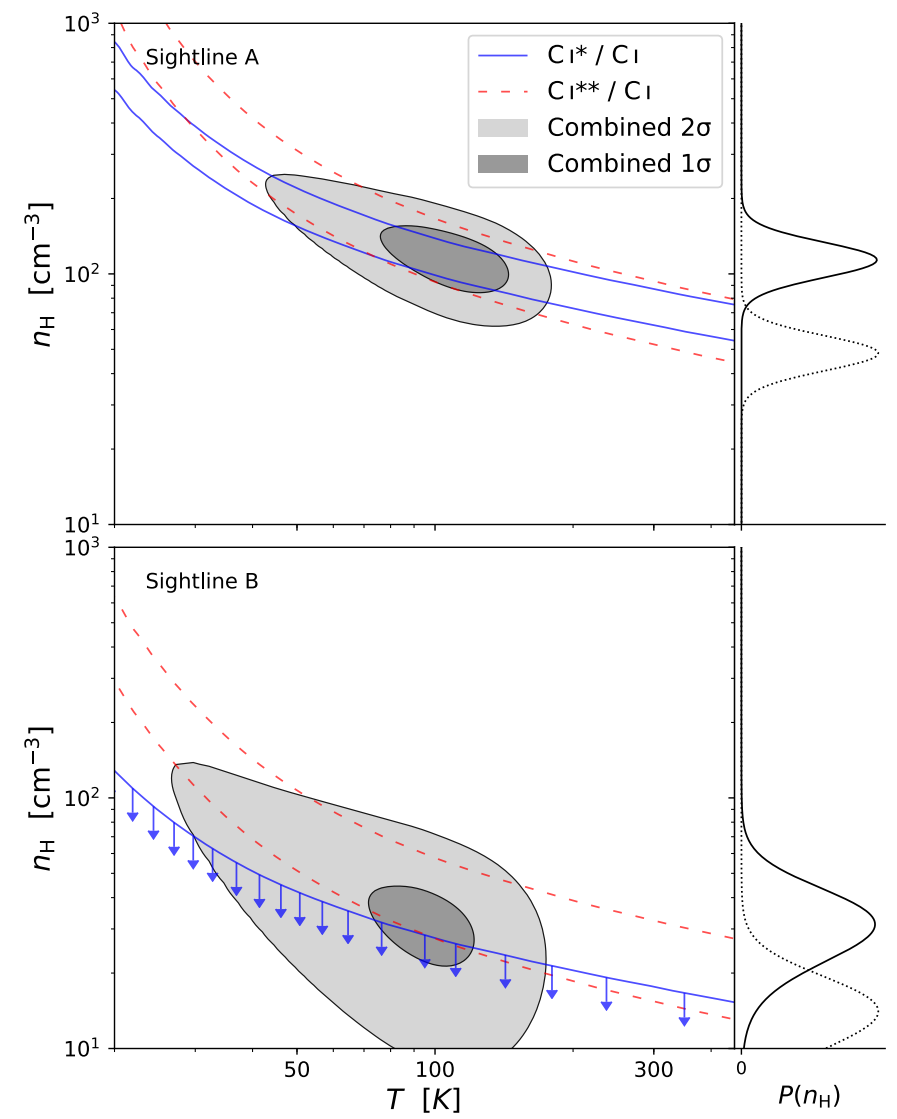

Fig. 8. Density vs. temperature derived from the observed excited $\mathrm{C}_{\mathrm{I}}$ fine-structure levels in the absorber toward image A (top) and image $\mathrm{B}$ (bottom) assuming $f_{\mathrm{H}_{2}}=1$. The allowed $1 \sigma$ ranges calculated for $\mathrm{CI}^{*}$ and $\mathrm{CI}^{* *}$ are shown as solid and dashed contours, respectively. The combined constraint on density and temperature is shown as the shaded gray regions. The curves on the right-hand axes show the marginalized probability density distributions for $n_{\mathrm{H}}$ assuming fully molecular gas (solid) or the observed, average molecular fraction (dotted).

Since we do not directly observe the absorbing galaxy behind the lensing galaxy, we have no prior information about the physical impact parameters of each line of sight through the absorbing galaxy. We therefore make the simplifying assumption that the two sightlines pierce the galaxy symmetrically at equal distances to the center. This allows us to infer a lower limit to the dynamical mass of $M_{\text {dyn }} \gtrsim 5 \times 10^{8} M_{\odot}$.

This is similar to the case of molecular absorption detected in a lensing galaxy at $z=0.76$ toward the quasar PMN 0134-0931 presented by Wiklind et al. (2018). The authors find that the molecular gas in the different lines of sight shows velocity offsets of $\sim 200 \mathrm{~km} \mathrm{~s}^{-1}$. Moreover, the lower limit to the dynamical mass is consistent with measurements of stellar masses of DLAs at similar redshifts (Christensen et al. 2014), which similarly serve as lower limits to the total dynamical mass.

\subsection{Filling factor}

Expanding on the scenario laid out above, we can constrain the volume filling factor of the cold gas. We assume that the cold gas must be distributed over scales of at least one kpc, in order to observe $C_{\text {I }}$ absorption in both sightlines. This is consistent with the findings of Wiklind et al. (2018), who found molecular absorption spread out over $5 \mathrm{kpc}$ with a more or less uniform 

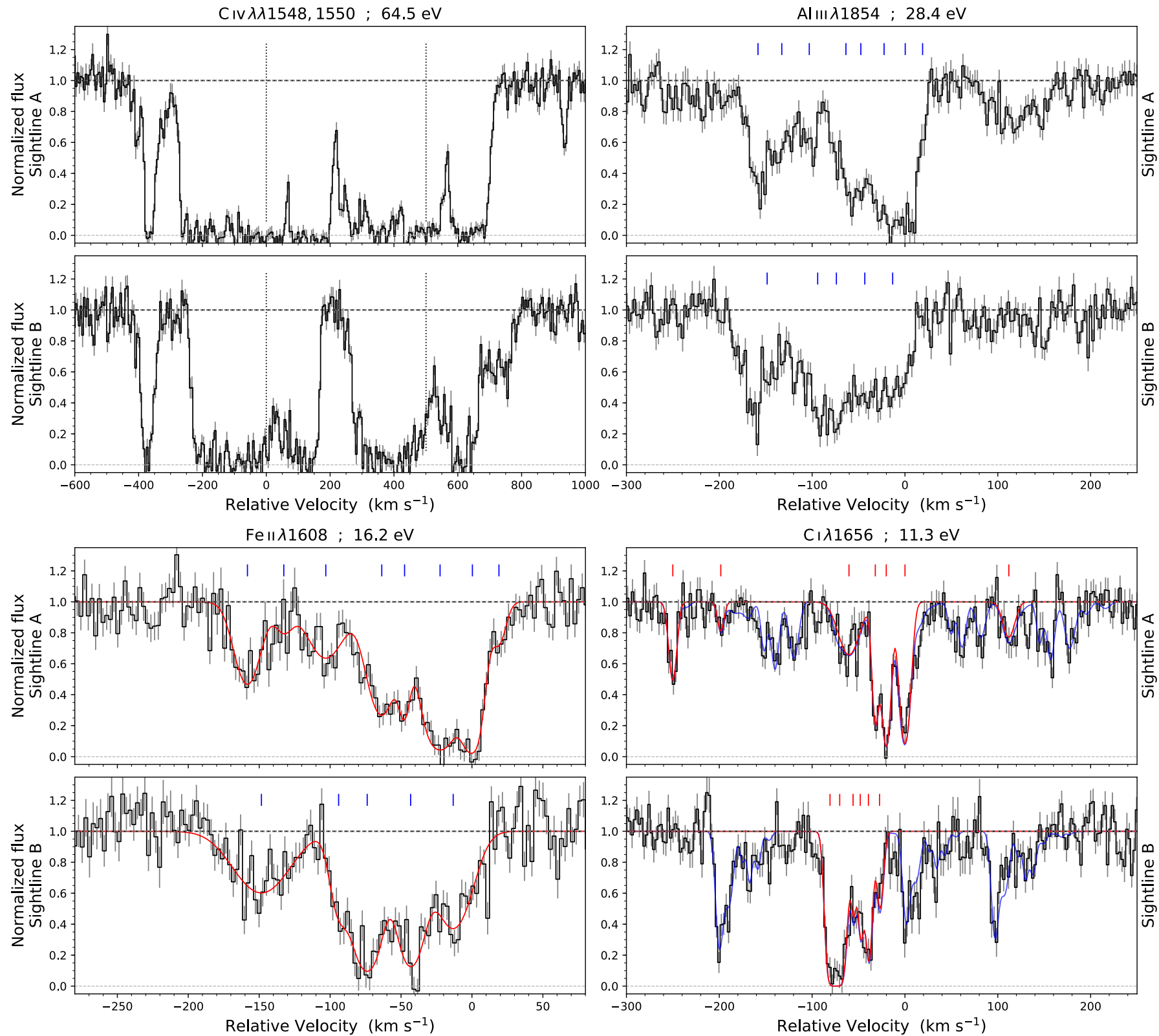

Fig. 9. Comparison of lines of various ionization states (the ionization potential is given for each ion) for the two sightlines. All panels show velocities relative to $z_{\text {sys }}=1.946938$. For $C_{\mathrm{I}}$ we show the total best-fit profile in blue, and we highlight the absorption from ground-state level in red (the red tick marks above show the location of the ground-state components). For Fe II, we show the best-fit profile in red and components as blue tick marks. For comparison, the same velocity components are shown for Al III. For the C IV doublet, we show only the zero-velocities as dotted vertical lines. The data have been rebinned by a factor of 2 for visual clarity.

coverage (Carilli \& Walter 2013, see also). In the following, we estimate the volume filling factor, assuming that the separation between the two sightlines is a representative scale over which the cold gas is found. This will result in an upper limit to the filling factor, as the cold gas might be spread over larger scales. Nonetheless, it is an instructive calculation that places direct constraints on the presence of cold gas in high-redshift galaxies.

We calculate the volume filling factor as the ratio

$f_{\mathrm{vol}}=\frac{N v}{V}$

where $N$ is the total number of clouds in the volume $V$ and $v$ is the volume of each cloud. We can also write $v$ as the product of the cross section, $\sigma$, and typical absorption length, $l$. Alternatively, we can write the average number of detected clouds along a line of sight as

$\mathcal{N}=\sigma L \frac{N}{V}$

where $L$ is the total absorption length along the line of sight through the medium. Substituting the unknown cloud number density $(N / V)$ in Eq. (3) using $\mathcal{N}$, we arrive at

$f_{\mathrm{vol}}=\mathcal{N} \frac{l}{L}$.

In order to estimate the individual cloud absorption length, $l$, we can use the physical density derived above: 
$l=\frac{N_{\mathrm{H}_{2}}}{n_{\mathrm{H}_{2}} \mathcal{N}}=\frac{2 N_{\mathrm{H}_{2}}}{n_{\mathrm{H}} \mathcal{N}}$

Here we assume that the bulk of the molecular absorption arises from the cold gas phase where the molecular fraction is close to unity, hence $n_{\mathrm{H}_{2}}=n_{\mathrm{H}} / 2$. We derive similar cloud sizes (to within a factor of two) for both lines of sight: $l \approx 0.1 \mathrm{pc}$. This further strengthens our assumption that the conditions are similar for the various cold clouds.

We neglected geometrical effects and used the physical separation of the two sightlines at the redshift of the absorber, $d_{\text {abs }}=0.7 \mathrm{kpc}$, as an estimate of the absorption length along the line of sight, since there is no reason to assume that the absorbing medium should be preferentially elongated along the line of sight nor perpendicular to it. Thus we assumed $L \geq d_{\text {abs }}$. This is a strict lower limit, as the $\mathrm{C}_{\mathrm{I}}$-bearing gas is most likely distributed over larger scales both along the line of sight and perpendicular to it.

We used the number of observed velocity components in the $\mathrm{C}_{\text {I }}$ profile as an estimate of the number of cold clouds. However, the number might be larger if some components are not resolved. We find an average of $\mathcal{N}=5$ cold clumps for both lines of sight, which translates into a volume filling factor of $f_{\mathrm{vol}}<0.002$. This is lower by almost an order of magnitude than the typical values of the CNM in the Milky Way ISM (Ferrière 2001, e.g.,).

Even though the volume filling factor is small, the cold gas is spread out over kpc scales, and hence the projected covering fraction, that is, the probability of intersecting the cold gas, becomes high, as evidenced by the detection of significant cold gas absorption in both lines of sight separated by $\sim 1 \mathrm{kpc}$.

While the covering fraction of cold, neutral gas may be high in the central parts of high-metallicity systems (where the pressure is high enough to allow efficient cooling), the bulk of the neutral gas in the overall population is dominated by the diffuse and warm gas spread over larger physical scales, as only $\sim 1 \%$ of $\mathrm{H}_{\mathrm{I}}$ absorbers show significant $\mathrm{C}_{\mathrm{I}}$ absorption (Ledoux et al. 2015). We can illustrate this scenario by a simple model in which the cross section of DLAs and $C_{I}$ absorbers is uniform within some typical physical scale. For DLAs at $z \sim 2$, this typical scale is $R_{\mathrm{HI}} \sim 10 \mathrm{kpc}$, but it may extend up to $\sim 30 \mathrm{kpc}$ or larger in a few cases (Rubin et al. 2015). Consequently, in order to match the 100 times lower incidence of $\mathrm{C}$ I absorbers, the typical scale over which $\mathrm{C}_{\mathrm{I}}$ bearing gas is detected must be $\sim 10$ times smaller. Hence, $R_{\mathrm{CI}}$ is on the order a few kpc. This typical scale for cold gas absorption of a few kpc is in good agreement with the constraint inferred from the observations presented here, $R_{\mathrm{CI}}>1 \mathrm{kpc}$. Taking into account the observed increase in $\mathrm{C}_{\mathrm{I}}$ incidence toward lower redshift (Ledoux et al. 2015), we qualitatively match the larger $R_{\mathrm{C}_{\mathrm{I}}} \gtrsim 5 \mathrm{kpc}$ inferred at intermediate redshifts of $z=0.76$ (Wiklind et al. 2018).

It is important to keep in mind that $\mathrm{C}_{\mathrm{I}}$ as a tracer of the cold gas is biased toward high-metallicity systems through the high abundance of carbon and through more efficient cooling and dust shielding in high-metallicity gas. Therefore, the incidence of cold gas is likely higher than that inferred purely by the incidence of $\mathrm{C}_{\mathrm{I}}$ absorption. This matches the conclusion by Neeleman et al. (2015) and Balashev \& Noterdaeme (2018) well, who found that the fraction of DLAs with significant amounts of cold gas is about $5 \%$ for a metallicity-unbiased sample.

Our study therefore suggests that the inferred volume filling factor of $\sim 0.1 \%$ reproduces the observed incidence of cold gas well, assuming a rather uniform cross section of cold gas on kpc scales immersed within a more widely spread warm, neutral gas phase extended on scales up to $10-30 \mathrm{kpc}$. This will provide important constraints for future numerical simulations designed to directly resolve the cold neutral and diffuse molecular phases of the ISM in high-redshift galaxies.

\section{Summary}

We here reported the analysis of the $z_{\mathrm{abs}}=1.9469$ absorber seen toward both images of the lensed quasar J1442+4055 at $z_{\mathrm{QSO}}=2.590$. We obtained imaging data from the Nordic Optical Telescope in the $r$ band and combined these data with archival SDSS imaging data in the $u, g, r, i$, and $z$ bands. After subtracting the PSF contribution from the quasar images, we detected the lensing galaxy in all filters except for the $u$ band. The higher quality $r$-band image from the NOT allowed us to constrain the structural parameters, while the larger filter coverage of the SDSS photometry enabled us to obtain a photometric redshift of $z_{\text {phot }}=0.35 \pm 0.15$.

We were able to recover the lensing galaxy spectrum by fitting the two LRIS spectra (covering wavelengths from 3600-8600 ^) with a combined quasar and galaxy model. The contribution of the lensing galaxy flux in each spectrum of the quasar was constrained from the NOT imaging data taken during similar conditions as the spectra. The recovered lens galaxy spectrum suffers from artifacts because the quasar emission lines and continuum vary. However, we clearly detect the $4000 \AA$ break together with $\mathrm{Ca}$ II, $\mathrm{Mg}$ I, and $\mathrm{Na}$ I absorption lines. The best-fit redshift of the lensing galaxy is $z_{\mathrm{L}}=0.284 \pm 0.001$. Using the lens redshift, we obtain a physical line-of-sight separation at the absorber redshift of $d_{\mathrm{abs}}=0.7 \mathrm{kpc}$.

We detect several metal species from different ionization stages in absorption from the $z_{\mathrm{abs}}=1.9469$ absorber. Moreover, we detect both neutral and molecular hydrogen absorption along both lines of sight. The inferred column densities of metals and hydrogen give consistent dust-corrected metallicities along both sightlines of $[\mathrm{S} / \mathrm{H}]=0.3 \pm 0.1$. The detection of strong absorption from $\mathrm{H}_{2}, \mathrm{CI}$, and its fine-structure levels indicates the presence of cold gas along both lines of sight.

The excitation of the lowest rotational levels of $\mathrm{H}_{2}$ provides a measure of the kinetic temperature of the gas phase harboring molecules and $\mathrm{C}_{\mathrm{I}}$. For both lines of sight, we infer consistent temperature estimates of $T_{A}=109 \pm 20$ and $T_{B}=89 \pm 25 \mathrm{~K}$. Using the estimated temperatures together with the observed fine-structure levels of neutral carbon, we modeled the gas cloud density using Popratio. The inferred average densities for the cold gas phase along the two lines of sight are $n_{\mathrm{H}}^{\mathrm{A}} \approx 110 \mathrm{~cm}^{-3}$ and $n_{\mathrm{H}}^{\mathrm{B}} \approx 40 \mathrm{~cm}^{-3}$.

We find that the low-ionization species (e.g., Fe II and Si II) exhibit similar velocity widths with numerous components. The relative strength of the various components differ for each sightline. The neutral absorption lines correlate with the strongest component of the low-ionization species. The neutral absorption in sightline B exhibits a velocity offset of $\sim 100 \mathrm{~km} \mathrm{~s}^{-1}$ relative to the absorption in sightline A. If we interpret this velocity offset as a pure result of rotation in an ordered disk, we obtain a lower limit to the system's dynamical mass of $M_{\text {dyn }} \gtrsim 5 \times 10^{8} M_{\odot}$.

Using the physical densities to infer the typical size of cold gas clouds $(l \sim 0.1 \mathrm{pc})$, we infer an upper limit to the volume filling factor of cold gas in this galaxy assuming that the cold gas is distributed over at least $0.7 \mathrm{kpc}$ corresponding to the line-of-sight separation. This yields a volume filling factor of $f_{\mathrm{vol}}<0.1 \%$.

We argue that the observations are consistent with a picture in which cold gas (as probed by neutral carbon) is confined in small clouds (with sizes of sub-pc to a few pc) that are distributed 
over kpc scales in high-redshift galaxies. This cold gas phase of $T \sim 100 \mathrm{~K}$ is immersed in a warmer $\left(T \sim 10^{4} \mathrm{~K}\right)$ gas phase, which can in turn extend up to a few tens of $\mathrm{kpc}$, with neutral hydrogen probing both these phases. This simple picture is able to explain the observed incidence rate of DLAs and $C_{I}$ absorbers.

Acknowledgements. We wish to thank the anonymous referee whose constructive comments helped improve the lens model significantly. The research leading to these results has received funding from the French Agence Nationale de la Recherche under grant no ANR-17-CE31-0011-01 (project "HIH2" - PI Noterdaeme). MF acknowledges support by the Science and Technology Facilities Council [grant number ST/P000541/1]. This project has received funding from the European Research Council (ERC) under the European Union's Horizon 2020 research and innovation programme (grant agreement No 757535). The Cosmic Dawn Center is funded by the Danish National Research Foundation. S.B. is supported by RSF grant 18-12-00301. F. C. acknowledges support from the Swiss National Science Foundation (SNSF). M.R. acknowledges support by a NASA Keck PI Data Award, administered by the NASA Exoplanet Science Institute. Based on observations made with the Nordic Optical Telescope, operated on the island of La Palma jointly by Denmark, Finland, Iceland, Norway, and Sweden, in the Spanish Observatorio del Roque de los Muchachos of the Instituto de Astrofísica de Canarias. Some of the data presented herein were obtained at the W. M. Keck Observatory from telescope time allocated to the National Aeronautics and Space Administration through the agency's scientific partnership with the California Institute of Technology and the University of California The Observatory was made possible by the generous financial support of the W. M. Keck Foundation. The authors wish to recognize and acknowledge the very significant cultural role and reverence that the summit of Maunakea has always had within the indigenous Hawaiian community. We are most fortunate to have the opportunity to conduct observations from this mountain.

\section{References}

Balashev, S. A., \& Noterdaeme, P. 2018, MNRAS, 478, L7

Balashev, S. A., Klimenko, V. V., Ivanchik, A. V., et al. 2014, MNRAS, 440, 225 Barnes, L. A., Garel, T., \& Kacprzak, G. G. 2014, PASP, 126, 969

Berg, T. A. M., Ellison, S. L., Prochaska, J. X., Venn, K. A., \& DessaugesZavadsky, M. 2015, MNRAS, 452, 4326

Bialy, S., \& Sternberg, A. 2016, ApJ, 822,

Brammer, G. B., van Dokkum, P. G., \& Coppi, P. 2008, ApJ, 686, 1503

Caffau, E., Bonifacio, P., Faraggiana, R., et al. 2005, A\&A, 441, 533

Carilli, C. L., \& Walter, F. 2013, ARA\&A, 51, 105

Carswell, R. F., Becker, G. D., Jorgenson, R. A., Murphy, M. T., \& Wolfe, A. M. 2012, MNRAS, 422, 1700

Christensen, L., Møller, P., Fynbo, J. P. U., \& Zafar, T. 2014, MNRAS, 445, 225

Churchill, C. W., Mellon, R. R., Charlton, J. C., \& Vogt, S. S. 2003, ApJ, 593, 203

Cooke, R., Pettini, M., Steidel, C. C., et al. 2010, MNRAS, 409, 679

Cooke, R. J., Pettini, M., \& Jorgenson, R. A. 2015, ApJ, 800, 12

Curran, S. J., Tzanavaris, P., Darling, J. K., et al. 2010, MNRAS, 402, 35

De Cia, A., Ledoux, C., Mattsson, L., et al. 2016, A\&A, 596, A97

Dutta, R., Srianand, R., Gupta, N., et al. 2017, MNRAS, 465, 4249

Ellison, S. L., Hennawi, J. F., Martin, C. L., \& Sommer-Larsen, J. 2007, MNRAS, 378, 801

Ferrière, K. M. 2001, Rev. Mod. Phys., 73, 1031

Field, G. B., Goldsmith, D. W., \& Habing, H. J. 1969, ApJ, 155, L149

Fioc, M., \& Rocca-Volmerange, B. 1997, A\&A, 326, 950

Fitzpatrick, E. L., \& Massa, D. 2007, ApJ, 663, 320

Gupta, N., Srianand, R., Petitjean, P., Noterdaeme, P., \& Saikia, D. J. 2009, MNRAS, 398, 201
Gupta, N., Srianand, R., Petitjean, P., et al. 2012, A\&A, 544, A21

Habing, H. J. 1968, Bull. Astron. Inst. Neth., 19, 421

Hennawi, J. F., Prochaska, J. X., Burles, S., et al. 2006, ApJ, 651, 61

Hennawi, J. F., Myers, A. D., Shen, Y., et al. 2010, ApJ, 719, 1672

Howk, J. C., Wolfe, A. M., \& Prochaska, J. X. 2005, ApJ, 622, L81

Jorgenson, R. A., Wolfe, A. M., \& Prochaska, J. X. 2010, ApJ, 722, 460

Jorgenson, R. A., Murphy, M. T., Thompson, R., \& Carswell, R. F. 2014, MNRAS, 443, 2783

Kanekar, N., Carilli, C. L., Langston, G. I., et al. 2005, Phys Lett. Rev., 95

Kanekar, N., Prochaska, J. X., Smette, A., et al. 2014, MNRAS, 438, 2131

Kelvin, L. S., Driver, S. P., Robotham, A. S. G., et al. 2012, MNRAS, 421, 1007

Khaire, V., \& Srianand, R. 2018, MNRAS, submitted [arXiv:1801.09693]

Krawczyk, C. M., Richards, G. T., Gallagher, S. C., et al. 2015, AJ, 149, 203

Krogager, J. K. 2018, ArXiv e-prints [arXiv:1803.01187]

Krumholz, M. R. 2012, ApJ, 759, 9

Krumholz, M. R., McKee, C. F., \& Tumlinson, J. 2009, ApJ, 699, 850

Ledoux, C., Petitjean, P., \& Srianand, R. 2003, MNRAS, 346, 209

Ledoux, C., Noterdaeme, P., Petitjean, P., \& Srianand, R. 2015, A\&A, 580, A8

Madau, P., Haardt, F., \& Rees, M. J. 1999, ApJ, 514, 648

Michalitsianos, A. G., Dolan, J. F., Kazanas, D., et al. 1997, ApJ, 474, 598

More, A., Oguri, M., Kayo, I., et al. 2016, MNRAS, 456, 1595

Murga, M., Zhu, G., Ménard, B., \& Lan, T.-W. 2015, MNRAS, 452, 511

Neeleman, M., Prochaska, J. X., \& Wolfe, A. M. 2015, ApJ, 800, 7

Noterdaeme, P., Ledoux, C., Petitjean, P., \& Srianand, R. 2008, A\&A, 481, 327

Noterdaeme, P., López, S., Dumont, V., et al. 2012, A\&A, 542, L33

Noterdaeme, P., Ledoux, C., \& Zou, S. et al. 2018, A\&A, 612, A58

Oguri, M., \& Marshall, P. J. 2010, MNRAS, 405, 2579

Oke, J. B., Cohen, J. G., Carr, M., et al. 1995, PASP, 107, 375

O'Meara, J. M., Lehner, N., Howk, J. C., et al. 2015, AJ, 150, 111

Peng, C. Y., Ho, L. C., Impey, C. D., \& Rix, H.-W. 2002, AJ, 124, 266

Petitjean, P., Srianand, R., \& Ledoux, C. 2000, A\&A, 364, L26

Planck Collaboration XVI. 2014, A\&A, 571, A16

Prochaska, J. X., Hennawi, J. F., \& Lee, K. G. et al. 2013, ApJ, 776, 136

Rafelski, M., Wolfe, A. M., Prochaska, J. X., Neeleman, M., \& Mendez, A. J. 2012, ApJ, 755, 89

Roy, N., Chengalur, J. N., \& Srianand, R. 2006, MNRAS, 365, L1

Rubin, K. H. R., Hennawi, J. F., Prochaska, J. X., et al. 2015, ApJ, 808, 38

Savage, B. D., \& Sembach, K. R. 1996, ARA\&A, 34, 279

Selsing, J., Fynbo, J. P. U., Christensen, L., \& Krogager, J.-K. 2016, A\&A, 585, A87

Sergeyev, A. V., Zheleznyak, A. P., Shalyapin, V. N., \& Goicoechea, L. J. 2016, MNRAS, 456, 1948

Sérsic, J. L. 1963, Boletin de la Asociacion Argentina de Astronomia La Plata Argentina, 6, 41

Silva, A. I., \& Viegas, S. M. 2001, Comput. Phys. Commun., 136, 319

Smette, A., Surdej, J., Shaver, P. A., et al. 1992, ApJ, 389, 39

Smette, A., Robertson, J. G., Shaver, P. A., et al. 1995, A\&As, 113, 199

Srianand, R., Petitjean, P., Ledoux, C., Ferland, G., \& Shaw, G. 2005, MNRAS, 362,549

Srianand, R., Gupta, N., Petitjean, P., et al. 2012, MNRAS, 421, 651

Vogt, S. S., Allen, S. L., \& Bigelow, B. C. et al. 1994, in Instrumentation in Astronomy VIII, 2198, 362

Wambsganss, J., \& Paczynski, B. 1991, AJ, 102, 864

Wiklind, T., \& Combes, F. 1995, A\&A, 299, 382

Wiklind, T., \& Combes, F. 1996, A\&A, 315, 86

Wiklind, T., Combes, F., \& Kanekar, N. 2018, ApJ, 864, 73

Wild, V., \& Hewett, P. C. 2005, MNRAS, 361, L30

Wolfe, A. M., Turnshek, D. A., Smith, H. E., \& Cohen, R. D. 1986, ApJs, 61, 249

Wolfe, A. M., Prochaska, J. X., \& Gawiser, E. 2003, ApJ, 593, 215

Wolfe, A. M., Prochaska, J. X., Jorgenson, R. A., \& Rafelski, M. 2008, ApJ, 681, 881

Wolfire, M. G., Hollenbach, D., McKee, C. F., Tielens, A. G. G. M., \& Bakes, E. L. O. 1995, ApJ, 443, 152 


\section{Appendix A: Absorption line fits}

We here provide the best-fit parameters for the individual components of the Voigt profile models for low-ionization species (Tables A.1 and A.2). In Fig. A.2 we show the best-fit profiles of the low-ionization transitions.
The best-fit parameters for the various rotational levels of $\mathrm{H}_{2}$ lines for the two lines of sight are given in Tables A.3 and A.4, and in Tables A.5 and A.6 we give the best-fit parameters for the $\mathrm{C}_{\text {I }}$ fine-structure lines for the two lines of sight. The best-fit line profiles for the $\mathrm{C}_{\mathrm{I}}$ complexes used to constrain the model are shown in Figure A.1.

Table A.1. Best-fit Voigt profile parameters for individual components of singly ionized metal species along sightline A.

\begin{tabular}{ccccc}
\hline \hline \multirow{2}{*}{$\begin{array}{c}\text { Rel. velocity } \\
\left(\mathrm{km} \mathrm{s}^{-1}\right)\end{array}$} & $\begin{array}{c}b \\
\left(\mathrm{~km} \mathrm{~s}^{-1}\right)\end{array}$ & Fe II & $\log \left(N / \mathrm{cm}^{-2}\right)$ \\
\hline$-158.3 \pm 0.8$ & $11.3 \pm 1.1$ & $13.81 \pm 0.04$ & $14.38 \pm 0.11$ & $14.29 \pm 0.06$ \\
$-132.6 \pm 1.1$ & $7.7 \pm 1.8$ & $13.09 \pm 0.19$ & $13.45 \pm 0.52$ & $13.82 \pm 0.08$ \\
$-103.0 \pm 1.2$ & $16.7 \pm 2.7$ & $13.74 \pm 0.06$ & $14.07 \pm 0.13$ & $14.00 \pm 0.06$ \\
$-63.6 \pm 1.2$ & $12.3 \pm 1.4$ & $14.08 \pm 0.05$ & $14.36 \pm 0.07$ & $14.84 \pm 0.06$ \\
$-47.5 \pm 0.8$ & $3.8 \pm 1.6$ & $13.63 \pm 0.12$ & $13.90 \pm 0.20$ & $14.30 \pm 0.19$ \\
$-22.5 \pm 0.9$ & $13.3 \pm 1.3$ & $14.49 \pm 0.05$ & $15.23 \pm 0.04$ & $15.12 \pm 0.05$ \\
$0.4 \pm 0.7$ & $7.3 \pm 0.7$ & $14.34 \pm 0.08$ & $14.95 \pm 0.07$ & $14.94 \pm 0.07$ \\
$19.0 \pm 0.0$ & $5.4 \pm 1.1$ & $13.17 \pm 0.10$ & $<14.07$ & $13.53 \pm 0.08$ \\
\hline
\end{tabular}

Notes. ${ }^{(a)}$ Relative to $z_{\text {sys }}=1.946938$.

Table A.2. Best-fit Voigt profile parameters for individual components of singly ionized metal species along sightline B.

\begin{tabular}{ccccc}
\hline \hline \multirow{2}{*}{$\begin{array}{c}\text { Rel. velocity } \\
\left(\mathrm{km} \mathrm{s}^{\mathrm{a}}\right)\end{array}$} & $b$ & \multicolumn{3}{c}{$\log \left(N / \mathrm{cm}^{-2}\right)$} \\
\cline { 3 - 5 }$\left(\mathrm{km} \mathrm{s}^{-1}\right)$ & Fe II & $\mathrm{S}_{\text {II }}$ & Si II \\
\hline$-148.4 \pm 2.0$ & $25.2 \pm 3.5$ & $13.97 \pm 0.07$ & - & $14.32 \pm 0.07$ \\
$-94.0 \pm 0.0^{b}$ & $7.4 \pm 1.9$ & $13.61 \pm 0.11$ & $<11.78$ & $14.52 \pm 0.17$ \\
$-73.9 \pm 0.9$ & $12.0 \pm 1.4$ & $14.32 \pm 0.05$ & $15.50 \pm 0.08$ & $15.00 \pm 0.08$ \\
$-43.2 \pm 1.1$ & $10.7 \pm 1.8$ & $14.22 \pm 0.06$ & $14.81 \pm 0.10$ & $14.45 \pm 0.20$ \\
$-13.2 \pm 1.9$ & $15.8 \pm 1.9$ & $14.06 \pm 0.06$ & $14.66 \pm 0.12$ & $14.64 \pm 0.11$ \\
\hline
\end{tabular}

Notes. ${ }^{(a)}$ Relative to $z_{\text {sys }}=1.946938 .{ }^{(b)}$ The redshift was kept fixed to the value obtained from a fit to Fe II only.
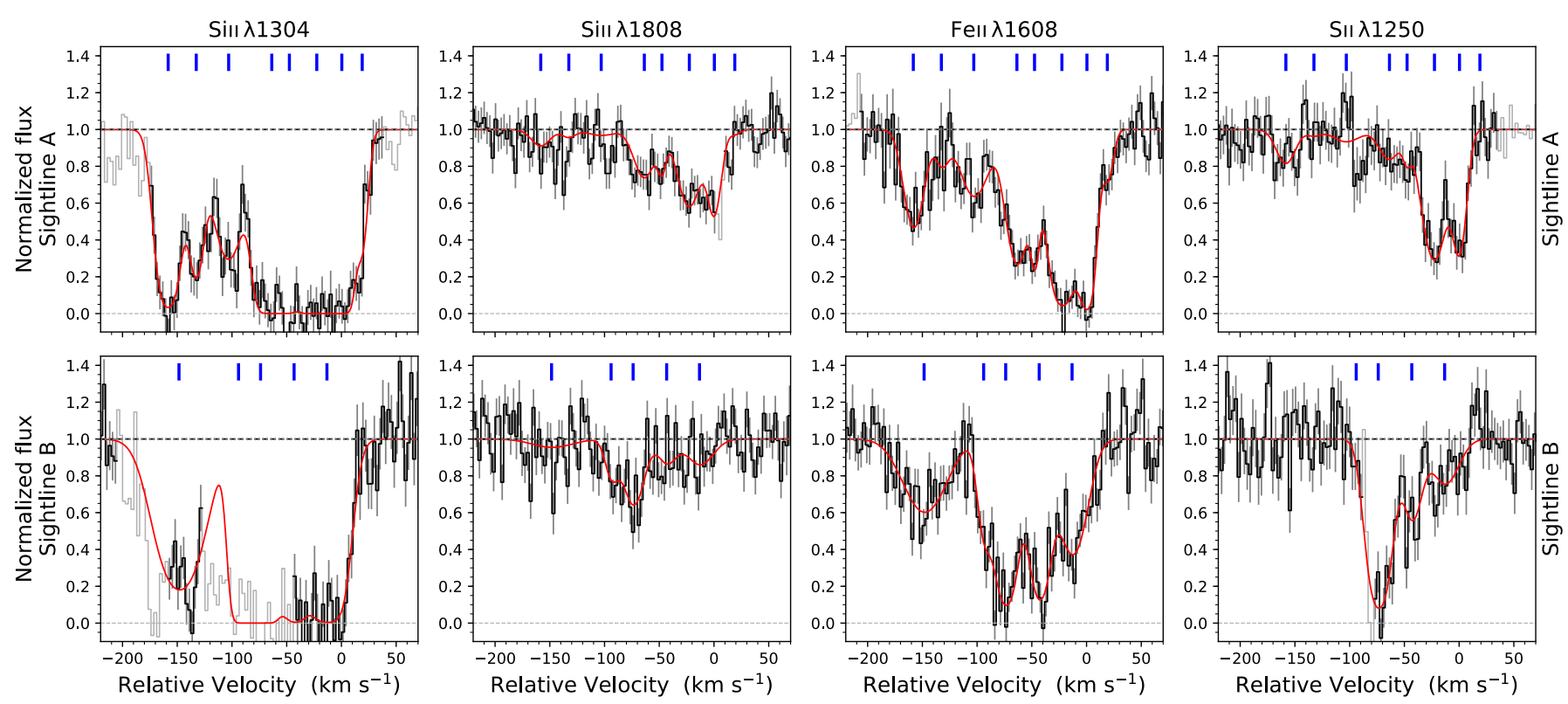

Fig. A.1. Comparison of $\mathrm{C}_{\mathrm{I}}$ fine-structure complexes for the two lines of sight. Regions of the spectra used to constrain the model are shown in black (gray regions were masked out during the fit). The best-fit profiles are shown in red, and the tick marks below the spectra indicate the positions of the individual components of the $J=0$ (blue solid), $J=1$ (red dashed), and $J=2$ (black dotted) transitions. The red tick marks above the spectra mark the location of weak intervening C IV systems. 

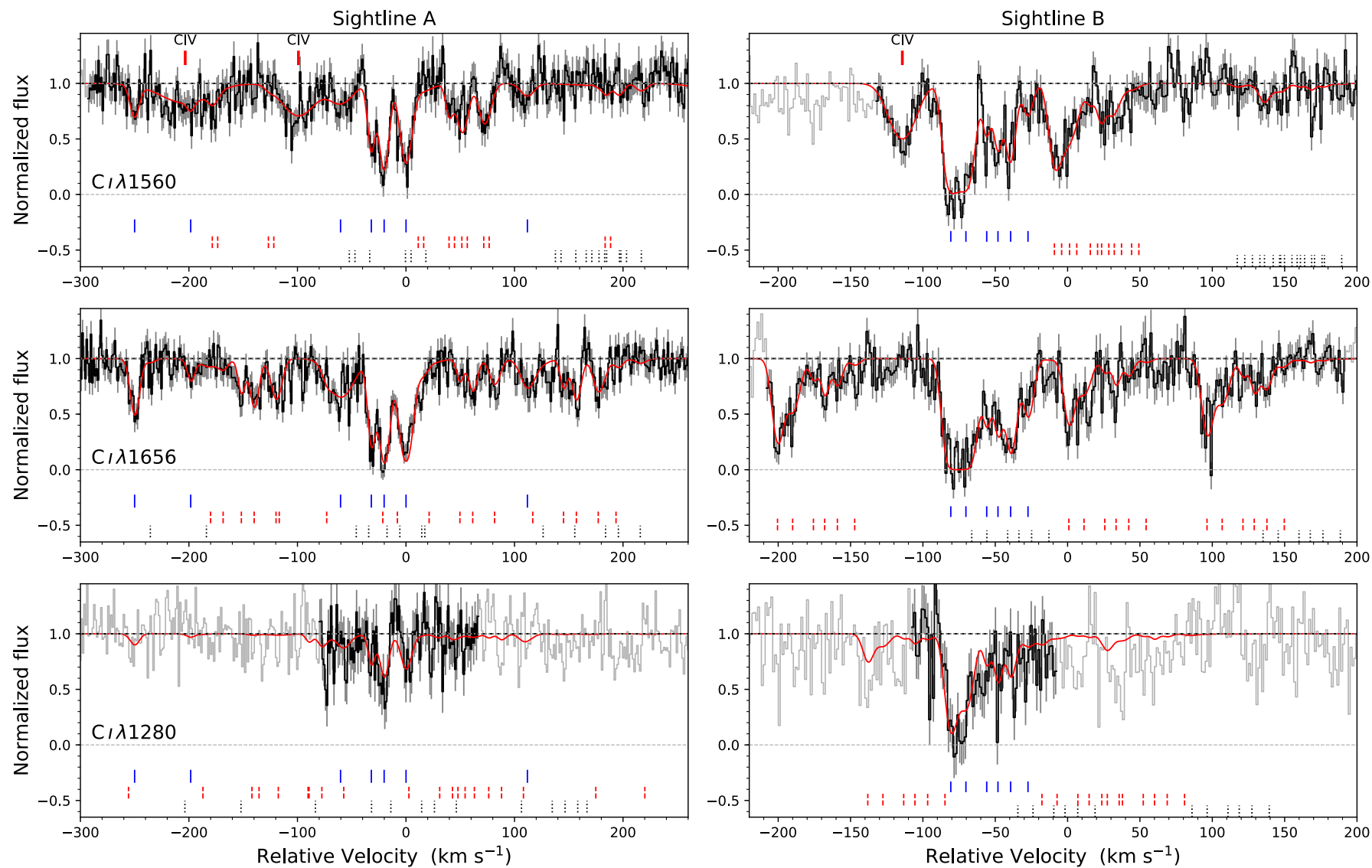

Fig. A.2. Comparison of singly ionized species for the two lines of sight. Regions of the spectra used to constrain the Voigt profile fitting are shown in black (gray regions were masked out during the fit due to Ly $\alpha$-forest contamination). The best-fit profiles are shown in red, and the blue tick marks above the spectra indicate the positions of the individual components.

Table A.3. Best-fit parameters for $\mathrm{H}^{2}$ for sightline A.

\begin{tabular}{cccc}
\hline \hline $\begin{array}{c}\text { Rel. velocity } \\
\left(\mathrm{km} \mathrm{s}^{-1}\right)\end{array}$ & $\begin{array}{c}b \\
\left(\mathrm{~km} \mathrm{~s}^{-1}\right)\end{array}$ & Rot. level & $\log \left(N / \mathrm{cm}^{-2}\right)$ \\
\hline-19 & 4.2 & $\mathrm{H}_{2}, J=0$ & $19.2 \pm 0.1$ \\
- & - & $\mathrm{H}_{2}, J=1$ & $19.4 \pm 0.1$ \\
- & - & $\mathrm{H}_{2}, J=2$ & $18.4 \pm 0.3$ \\
- & - & $\mathrm{H}_{2}, J=3$ & $18.7 \pm 0.2$ \\
\hline
\end{tabular}

Notes. ${ }^{(a)}$ Relative to $z_{\text {sys }}=1.946938$.

Table A.4. Best-fit parameters for $\mathrm{H}^{2}$ for sightline B.

\begin{tabular}{cccc}
\hline $\begin{array}{c}\text { Rel. velocity } \\
\left(\mathrm{km} \mathrm{s}^{-1}\right)\end{array}$ & $\begin{array}{c}b \\
\left(\mathrm{~km} \mathrm{~s}^{-1}\right)\end{array}$ & Rot. level & $\log \left(N / \mathrm{cm}^{-2}\right)$ \\
\hline-42 & 2.9 & $\mathrm{H}_{2}, J=0$ & $19.5 \pm 0.2$ \\
- & - & $\mathrm{H}_{2}, J=1$ & $19.6 \pm 0.2$ \\
- & - & $\mathrm{H}_{2}, J=2$ & $18.7 \pm 0.2$ \\
\hline
\end{tabular}

Notes. ${ }^{(a)}$ Relative to $z_{\text {sys }}=1.946938$. 
Table A.5. Best-fit parameters for $\mathrm{C}_{\mathrm{I}}$ for sightline A.

\begin{tabular}{ccccc}
\hline \hline \multirow{2}{*}{$\begin{array}{c}\text { Rel. velocity } \\
\left(\mathrm{km} \mathrm{s}^{-1}\right)\end{array}$} & $b$ & \multicolumn{3}{c}{$\log \left(\mathrm{N} / \mathrm{cm}^{-2}\right)^{b}$} \\
\cline { 3 - 5 }$\left(\mathrm{km} \mathrm{s}^{-1}\right)$ & $\mathrm{C}_{\mathrm{I}}$ & $\mathrm{C}^{*}$ & $\mathrm{C} \mathrm{I}^{* *}$ \\
\hline$-250.0 \pm 0.5$ & $4.4 \pm 0.8$ & $13.08 \pm 0.05$ & $12.68 \pm 0.06$ & $11.90 \pm 0.13$ \\
$-198.4 \pm 1.3$ & $3.0 \pm 2.9$ & $12.46 \pm 0.14$ & $12.06 \pm 0.15$ & $11.28 \pm 0.19$ \\
$-60.3 \pm 1.4$ & $14.4 \pm 1.9$ & $13.25 \pm 0.05$ & $12.85 \pm 0.06$ & $12.06 \pm 0.13$ \\
$-31.9 \pm 0.3$ & $2.5 \pm 0.2$ & $13.48 \pm 0.06$ & $13.08 \pm 0.05$ & $12.30 \pm 0.14$ \\
$-20.1 \pm 0.3$ & $4.2 \pm 0.4$ & $13.75 \pm 0.04$ & $13.35 \pm 0.03$ & $12.57 \pm 0.13$ \\
$0.0 \pm 0.3$ & $5.7 \pm 0.4$ & $13.71 \pm 0.03$ & $13.31 \pm 0.03$ & $12.53 \pm 0.12$ \\
$111.9 \pm 1.6$ & $7.8 \pm 2.4$ & $12.80 \pm 0.10$ & $12.40 \pm 0.10$ & $11.62 \pm 0.15$ \\
\hline$z$ & \multicolumn{4}{c}{} \\
\hline $1.96408 \pm 0.00001$ & $19.7 \pm 1.6$ & $13.50 \pm 0.03$ \\
$1.96800 \pm 0.00004$ & $31.2 \pm 6.2$ & $13.14 \pm 0.07$ \\
\hline
\end{tabular}

Notes. ${ }^{(a)}$ Relative to $z_{\text {sys }}=1.946938 .{ }^{(b)}$ The column densities for $\mathrm{C}{ }^{*}$ and $\mathrm{C}^{* *}$ were tied to the ground level assuming two freely varying column density ratios $r^{*}$ and $r^{* *}$ for all components.

Table A.6. Best-fit parameters for $\mathrm{C}_{\mathrm{I}}$ for sightline B.

\begin{tabular}{|c|c|c|c|c|}
\hline \multirow{2}{*}{$\begin{array}{l}\text { Rel. velocity } \\
\quad\left(\mathrm{km} \mathrm{s}^{-1}\right)\end{array}$} & \multirow{2}{*}{$\begin{array}{c}b \\
\left(\mathrm{~km} \mathrm{~s}^{-1}\right)\end{array}$} & \multicolumn{3}{|c|}{$\log \left(N / \mathrm{cm}^{-2}\right)^{b}$} \\
\hline & & $\mathrm{C}_{\mathrm{I}}$ & $\mathrm{CI}^{*}$ & $\mathrm{C}_{\mathrm{I}^{* *}}$ \\
\hline$-80.7 \pm 0.5$ & $3.6 \pm 0.4$ & $14.47 \pm 0.09$ & $13.75 \pm 0.06$ & $12.82 \pm 0.13$ \\
\hline$-70.3 \pm 1.0$ & $5.0 \pm 0.9$ & $14.16 \pm 0.09$ & $13.44 \pm 0.08$ & $12.51 \pm 0.14$ \\
\hline$-55.9 \pm 0.6$ & $1.1 \pm 0.2$ & $13.54 \pm 0.15$ & $12.82 \pm 0.13$ & $11.90 \pm 0.19$ \\
\hline$-48.1 \pm 0.4$ & $1.3 \pm 0.2$ & $13.88 \pm 0.12$ & $13.16 \pm 0.10$ & $12.23 \pm 0.15$ \\
\hline$-39.3 \pm 0.4$ & $2.6 \pm 0.1$ & $13.67 \pm 0.07$ & $12.95 \pm 0.08$ & $12.02 \pm 0.14$ \\
\hline$-27.4 \pm 0.7$ & $3.0 \pm 0.0$ & $13.01 \pm 0.08$ & $12.29 \pm 0.10$ & $11.37 \pm 0.15$ \\
\hline$z$ & $\begin{array}{c}b \\
\left(\mathrm{~km} \mathrm{~s}^{-1}\right)\end{array}$ & $\begin{array}{c}\log \left(N / \mathrm{cm}^{-2}\right) \\
C_{\text {IV }}\end{array}$ & & \\
\hline $1.963932 \pm 0.000006$ & $11.5 \pm 0.9$ & $13.58 \pm 0.03$ & & \\
\hline
\end{tabular}

Notes. ${ }^{(a)}$ Relative to $z_{\text {sys }}=1.946938 .{ }^{(b)}$ The column densities for $\mathrm{C}{ }^{*}$ and $\mathrm{C}{ }^{* *}$ were tied to the ground level assuming two freely varying column density ratios $r^{*}$ and $r^{* *}$ for all components. 